\title{
FAMILIES OF ELLIPTIC CURVES WITH RATIONAL TORSION POINTS OF EVEN ORDER
}

\author{
BORIS M. BEKKER AND YURI G. ZARHIN
}

\section{INTRODUCTION}

This paper is a follow up of [1. Our aim is to give an explicit construction of versal families of elliptic curves with a torsion point of a certain (small) order. The problem of constructing such families goes back to Beppo Levi [7, 8 , and is closely related to certain modular curves of genus zero. However, our approach based on the explicit formulas for "halves" of points on elliptic curves [1, Sect. 2] is quite elementary.

Here are our main results.

Theorem 1.1. Let $K$ be a field with $\operatorname{char} K \neq 2$. Let $E$ be an elliptic curve over $K$.

(1) Let a be a nonzero element of $K$. The following conditions are equivalent.

(4i) $E(K)$ contains exactly one point of order 2 and a point of order 4.

(4ii) There exists a nonzero element $b \in K$ such that $a^{2}+4 b$ is not a square in $K$ and $E$ is $K$-isomorphic to the elliptic curve

$$
\mathcal{E}_{a, b}^{(4)}: y^{2}=\left(x^{2}+\left(a^{2}+2 b\right) x+b^{2}\right) x .
$$

(2) The following conditions are equivalent.

(8i) $E(K)$ contains exactly one point of order 2 and a point of order 8.

(8ii) There exists $t \in K \backslash\{0, \pm 1\}$ such that $2 t^{2}-1$ is not a square in $K$ and $E$ is $K$-isomorphic to the elliptic curve

$$
\mathcal{E}_{t}^{(8)}: y^{2}=\left(x^{2}+2 \frac{t^{4}+2 t^{2}-1}{\left(t^{2}-1\right)^{2}} x+1\right) x .
$$

(3) The following conditions are equivalent.

(6i) $E(K)$ contains exactly one point of order 2 and a point of order 6.

(6ii) There exists $t \in K \backslash\{0,-4,1 / 2\}$ such that $t^{2}+4 t$ is not a square in $K$ and $E$ is $K$-isomorphic to the elliptic curve

$$
\mathcal{E}_{t}^{(6)}: y^{2}=\left(x^{2}+\left(t^{2}+2 t\right) x+t^{2}\right)(x+1) .
$$

(4) The following conditions are equivalent.

(12i) $E(K)$ contains exactly one point of order 2 and a point of order 12.

(12ii) There exists $t \in K \backslash\{0, \pm 1, \pm \sqrt{-1}\}$ such that $\left(t^{2}+1\right)\left(3 t^{2}-1\right)$ is not a square in $K, 3 t^{2}+1 \neq 0$, and $E$ is $K$-isomorphic to the elliptic curve

$$
\mathcal{E}_{t}^{(12)}: y^{2}=(x+1)\left(x^{2}+\frac{8 t^{2}\left(t^{2}+1\right)\left(t^{4}+4 t^{2}-1\right)}{\left(t^{2}-1\right)^{4}} x+\frac{16 t^{4}\left(t^{2}+1\right)^{2}}{\left(t^{2}-1\right)^{4}}\right) \text {. }
$$

(5) The following conditions are equivalent.

(10i) $E(K)$ contains exactly one point of order 2 and a point of order 10. 
(10ii) There exists $t \in K \backslash\{0, \pm 1,(-1 \pm \sqrt{5}) / 2,2 \pm \sqrt{5}\}$ such that $t\left(t^{2}+t-1\right)$ is not a square in $K$ and $E$ is $K$-isomorphic to the elliptic curve

$$
\mathcal{E}_{t}^{(10)}: y^{2}=(x+1)\left(x^{2}+\frac{8 t^{2}\left(t^{3}+t^{2}-t+1\right)}{(t-1)^{2}(t+1)^{4}} x+\frac{16 t^{4}}{(t-1)^{2}(t+1)^{4}}\right) .
$$

Remarks 1.2. (1) $\mathcal{E}_{a, b}^{(4)}(K)$ contains exactly two points of order 4, namely, $(-b, a b)$ and $(-b,-a b)$. On the other hand, dividing both sides of the equation for $\mathcal{E}_{a, b}^{(4)}$ by $a^{6}$ and introducing

$$
\tilde{x}=\frac{x}{a^{2}}, \tilde{y}=\frac{y}{a^{3}}, \tilde{b}=\frac{b}{a^{2}},
$$

we obtain that $\mathcal{E}_{a, b}^{(4)}$ is $K$-isomorphic to

$$
\mathcal{E}_{1, \tilde{b}}^{(4)}: \tilde{y}^{2}=\left(\tilde{x}^{2}+(1+2 \tilde{b}) \tilde{x}+\tilde{b}^{2}\right) \tilde{x} .
$$

(2) $\mathcal{E}_{t}^{(8)}(K)$ contains exactly two points of order 4 , namely,

$$
\left(1, \frac{2 t}{1-t^{2}}\right),\left(1,-\frac{2 t}{1-t^{2}}\right)
$$

and exactly four points of order 8 , namely,

$$
\left(\frac{1+t}{1-t}, \frac{-2 t}{(1-t)^{2}}\right),\left(\frac{1-t}{1+t}, \frac{2 t}{(1+t)^{2}}\right)
$$

and their negatives

$$
\left(\frac{1+t}{1-t}, \frac{2 t}{(1-t)^{2}}\right),\left(\frac{1-t}{1+t}, \frac{-2 t}{(1+t)^{2}}\right) .
$$

(3) $\mathcal{E}_{t}^{(6)}(K)$ contains the points $(0, t)$ and $(0,-t)$ of order 3 and the points $\left(-2 t, t-2 t^{2}\right)$ and $\left(-2 t, 2 t^{2}-t\right)$ of order 6 . If char $=3$, then there are no other points of order 3 or 6 in $\mathcal{E}_{t}^{(6)}(K)$.

(4) $\mathcal{E}_{t}^{(12)}(K)$ contains the points

$$
\left(0, \frac{4 t^{2}\left(t^{2}+1\right)}{\left(t^{2}-1\right)^{2}}\right),\left(0,-\frac{4 t^{2}\left(t^{2}+1\right)}{\left(t^{2}-1\right)^{2}}\right)
$$

of order 3 , and exactly two points of order 4, namely,

$$
\left(-\frac{3 t^{2}+1}{t^{2}-1},-\frac{8 t^{3}\left(3 t^{2}+1\right)}{\left(t^{2}-1\right)^{3}}\right),\left(-\frac{3 t^{2}+1}{t^{2}-1}, \frac{8 t^{3}\left(3 t^{2}+1\right)}{\left(t^{2}-1\right)^{3}}\right) .
$$

(5) $\mathcal{E}_{t}^{(10)}(K)$ contains a point $\left(0,4 t^{2} /(t-1)(t+1)^{2}\right)$ of order 5 and exactly one point $(-1,0)$ of order 2.

The paper is organized as follows. First three sections deal with division by 2 in $E(K)$ under various assumptions about the existence of $K$-points of order 2 on the elliptic curve $E$. Our goal is to obtain explicit formulas that will be used in the next five sections containing a construction of versal families of elliptic curves with rational points of order 4,8,6,12, and 10, respectively (and with exactly one rational point of order 2 ). The last section deals with versal families of (ordinary) elliptic curves in characteristic 2 that admit a rational point of order 4 or 8 . 
FAMILIES OF ELLIPTIC CURVES WITH RATIONAL TORSION POINTS OF EVEN ORDER3

\section{REVIEW OF [1], SECTION 2}

2.1. Let $K$ be a field with $\operatorname{char}(K) \neq 2$ and $\bar{K}$ an algebraic closure of $K$. Let $f(x) \in$ $K[x]$ be a monic cubic polynomial without repeated roots and $\left\{\alpha_{1}, \alpha_{2}, \alpha_{3}\right\} \subset \bar{K}$ the set of roots of $f(x)$. Clearly

$$
f(x)=\left(x-\alpha_{1}\right)\left(x-\alpha_{2}\right)\left(x-\alpha_{3}\right) \in \bar{K}[x] .
$$

Let

$$
E=E_{f}: y^{2}=f(x)
$$

be an elliptic curve over $K$ and $\infty$ its only infinite point (the zero of the group law).

We have

The points

$$
\begin{array}{r}
E(K)=\left\{\left(x_{0}, y_{0}\right) \in K^{2} \mid y_{0}^{2}=f\left(x_{0}\right)\right\} \coprod\{\infty\} \\
\subset\left\{\left(x_{0}, y_{0}\right) \in \bar{K}^{2} \mid y_{0}^{2}=f\left(x_{0}\right)\right\} \coprod\{\infty\}=E(\bar{K}) .
\end{array}
$$

are the only points of order 2 on $E$.

Remark 2.2. A root $\alpha_{i}$ lies in $K$ if and only if $W_{i}=\left(\alpha_{i}, 0\right) \in E(K)$. This implies that $E(K)$ contains exactly one point of order 2 if and only if $f(x)$ has exactly one root in $K$.

The following assertion is pretty well-known [2, 3] (see also [1]).

Theorem 2.3. Suppose that all the roots $\alpha_{i}$ of $f(x)$ lie in $K$, i.e., all three points of order 2 on $E$ lie in $E(K)$. Let $P=\left(x_{0}, y_{0}\right) \in E(K)$. Then $P \in 2 E(K)$ if and only if all $x_{0}-\alpha_{i}$ are squares in $K$. In addition, each point $Q \in E(\bar{K})$ with $2 Q=P$ actually lies in $E(K)$.

In what follows we discuss the divisibility by 2 of points in $E(K)$ when not necessarily all roots of $f(x)$ lie in $K$.

2.4. Let $P=\left(x_{0}, y_{0}\right) \in E(\bar{K})$. There are precisely four points $Q \in E(\bar{K})$ such that $2 Q=P$. The following explicit construction of all halves $Q$ 's was described in [1, Sect. 2]. Let us choose square roots

$$
r_{i}=\sqrt{x_{0}-\alpha_{i}} \in \bar{K}(1 \leq i \leq 3) .
$$

(There are exactly eight choices of such triples $\left(r_{1}, r_{2}, r_{3}\right)$.) We have

$$
r_{1} r_{2} r_{3}=\sqrt{\left(x_{0}-\alpha_{1}\right)\left(x_{0}-\alpha_{2}\right)\left(x_{0}-\alpha_{3}\right)}= \pm y_{0} \text {. }
$$

Since all $\alpha_{i}$ 's are distinct, $r_{i} \neq \pm r_{j}$ if $i \neq j$. This implies that for all $i \neq j$

$$
r_{i} \pm r_{j} \neq 0 \text {. }
$$

Let us consider only the triples that satisfy

$$
r_{1} r_{2} r_{3}=-y_{0} .
$$

(There are exactly four choices of such triples $\left(r_{1}, r_{2}, r_{3}\right)$.) Let us put

$$
s_{1}=s_{1}\left(r_{1}, r_{2}, r_{3}\right)=r_{1}+r_{2}+r_{3}, s_{2}=s_{2}\left(r_{1}, r_{2}, r_{3}\right)=r_{1} r_{2}+r_{2} r_{3}+r_{3} r_{1} .
$$

Then the point

$$
\begin{gathered}
Q=Q_{r_{1}, r_{2}, r_{3}}=\left(x_{1}, y_{1}\right):=\left(x_{0}+s_{2},-y_{0}-s_{1} s_{2}\right)= \\
\left(x_{0}+r_{1} r_{2}+r_{2} r_{3}+r_{3} r_{1},-\left(r_{1}+r_{2}\right)\left(r_{2}+r_{3}\right)\left(r_{3}+r_{1}\right)\right)
\end{gathered}
$$


lies in $E(\bar{K})$ and satisfies

$$
2 Q=Q_{r_{1}, r_{2}, r_{3}}=P \in E(\bar{K}) .
$$

In addition,

$$
l:=-s_{1}=-\left(r_{1}+r_{2}+r_{3}\right)
$$

is the slope of the tangent line $\mathcal{L}_{Q}$ to $E$ at $Q$ [1, Proof of Th. 2.1]. More precisely, $\mathcal{L}_{Q}$ passes through the point $-P=\left(x_{0},-y_{0}\right)$ and is defined by the equation

$$
y=l\left(x-x_{0}\right)-y_{0} .
$$

Distinct choices of triples $\left(r_{1}, r_{2}, r_{3}\right)$ give rise to distinct halves $Q_{r_{1}, r_{2}, r_{3}}$ 's. More precisely [1, Th. 2.3],

$Q_{r_{1},-r_{2},-r_{3}}=Q_{r_{1}, r_{2}, r_{3}}+W_{1}, Q_{-r_{1}, r_{2},-r_{3}}=Q_{r_{1}, r_{2}, r_{3}}+W_{2}, Q_{-r_{1},-r_{2}, r_{3}}=Q_{r_{1}, r_{2}, r_{3}}+W_{3}$.

(That is how we get all four $Q \in E(\bar{K})$ with $2 Q=P$.) Conversely, if we are given $Q=\left(x_{1}, y_{1}\right) \in E(\bar{K})$, then $Q=Q_{r_{1}, r_{2}, r_{3}}$ with

$$
r_{i}=-\frac{y_{1}}{2} \cdot\left(-\frac{1}{x_{1}-\alpha_{i}}+\frac{1}{x_{1}-\alpha_{j}}+\frac{1}{x_{1}-\alpha_{k}}\right)
$$

for each permutation $i, j, k$ of $1,2,3$.

Formulas from Sect. 2.4 almost immediately lead to the following statement.

Theorem 2.5. Suppose that $P=\left(x_{0}, y_{0}\right) \in E(K)$. Suppose that square roots $r_{i}=\sqrt{x_{0}-\alpha_{i}} \in \bar{K}(i=1,2,3)$ satisfy

$$
r_{1} r_{2} r_{3}=-y_{0} ; s_{1}=r_{1}+r_{2}+r_{3} \in K, s_{2}=r_{1} r_{2}+r_{2} r_{3}+r_{3} r_{1} \in K .
$$

Then the point $Q_{r_{1}, r_{2}, r_{3}} \in E(\bar{K})$ defined by formula (6) enjoys the following properties:

$$
Q_{r_{1}, r_{2}, r_{3}} \in E(K), 2 Q_{r_{1}, r_{2}, r_{3}}=P .
$$

Conversely, suppose that $Q \in E(K)$ satisfies $2 Q=P$. Then there exists precisely one triple $\left\{r_{1}, r_{2}, r_{3}\right\}$ of square roots $r_{i}=\sqrt{x_{0}-\alpha_{i}} \in \bar{K}(i=1,2,3)$ that satisfy (10) and such that $Q=Q_{r_{1}, r_{2}, r_{3}}$.

Proof. Suppose that the square roots $r_{i}(i=1,2,3)$ satisfy (10). This implies that both $s_{1}$ and $s_{2}$ defined in (5) lie in $K$. The point $Q_{r_{1}, r_{2}, r_{3}}=\left(x_{1}, y_{1}\right)$ defined by formula (6) lies in $E(\bar{K})$ and $2 Q=P$. Since, $x_{0}, y_{0}, s_{1}, s_{2} \in K$, the formulas (6) imply that $x_{1}, y_{1} \in K$, i.e., $Q \in E(K)$.

Conversely, suppose that $P=2 Q$ with $Q=\left(x_{1}, y_{1}\right) \in E(K)$. Then

$$
Q \in E(K) \subset E(\bar{K}) ; x_{1}, y_{1} \in K .
$$

There exists exactly one triple $\left\{r_{1}, r_{2}, r_{3}\right\}$ of square roots $r_{i}=\sqrt{x_{0}-\alpha_{i}} \in \bar{K}$ that satisfy (10) such that $r_{1} r_{2} r_{3}=-y_{0}$ and $Q=Q_{r_{1}, r_{2}, r_{3}}$. In light of (6), $s_{2}=x_{1}-x_{0}$. Since both $x_{0}, x_{1} \in K$, we obtain that $s_{2} \in K$. Since $Q \in E(K)$, the slope $l$ of the tangent line to $E$ at $Q$ lies in $K$. In light of (7), $l=-s_{1}$ and therefore $s_{1} \in K$. To summarize: $r_{1} r_{2} r_{3}=-y_{0}$ and $r_{1}+r_{2}+r_{3}=s_{1} \in K, s_{2} \in K$. In other words, the triple $\left\{r_{1}, r_{2}, r_{3}\right\}$ satisfies the conditions (10).

Corollary 2.6. Suppose that $P=\left(x_{0}, y_{0}\right) \in E(K)$. Then the following conditions are equivalent.

(i) $P \in 2 E(K)$, i.e., there exists $Q \in E(K)$ such that $2 Q=P$. 
FAMILIES OF ELLIPTIC CURVES WITH RATIONAL TORSION POINTS OF EVEN ORDER5

(ii) One may choose square roots $r_{i}=\sqrt{x_{0}-\alpha_{i}} \in \bar{K}$ in such a way that

$$
s_{1}=r_{1}+r_{2}+r_{3} \in K, s_{2}=r_{1} r_{2}+r_{2} r_{3}+r_{3} r_{1} \in K \text {. }
$$

(iii) One may choose square roots $r_{i}=\sqrt{x_{0}-\alpha_{i}} \in \bar{K}$ in such a way that $r_{1} r_{2} r_{3}=-y_{0} ; s_{1}=r_{1}+r_{2}+r_{3} \in K, s_{2}=r_{1} r_{2}+r_{2} r_{3}+r_{3} r_{1} \in K$.

Proof. By Theorem 2.5, one has only to check that (ii) implies (iii). Indeed, suppose we are given a triple $\left(r_{1}, r_{2}, r_{3}\right)$ of square roots $r_{i}=\sqrt{x_{0}-\alpha_{i}}$ such that

$$
s_{1}=r_{1}+r_{2}+r_{3} \in K, s_{2}=r_{1} r_{2}+r_{2} r_{3}+r_{3} r_{1} \in K \text {. }
$$

By equality (2), $r_{1} r_{2} r_{3}= \pm y_{0}$. Replacing if necessary $\left(r_{1}, r_{2}, r_{3}\right)$ by $\left(-r_{1},-r_{2},-r_{3}\right)$ (and $s_{1}$ by $-s_{1}$ ), we may and will assume that $r_{1} r_{2} r_{3}=-y_{0}$ and still $s_{1}, s_{2} \in K$. This proves that (ii) implies (actually equivalent to) (iii).

\section{Division BY 2}

In this section we discuss division by 2 in $E(K)$ when $E(K)$ contains exactly one point of order 2 .

Let $K$ be a field with $\operatorname{char}(K) \neq 2$. Let $\alpha$ be an element of $K$,

$$
g(x)=x^{2}+p x+q \in K[x]
$$

a monic irreducible quadratic polynomial (in particular, $\alpha$ is not a root of $g(x)$ ), and $K_{g}=K[x] / g(x) K[x]$ the corresponding quadratic field extension of $K$. We write $\mathbf{x}$ for the image of $x$ in $K_{g}$ with respect to the natural surjective homomorphism

$$
K[x] \rightarrow K[x] / g(x) K[x]=K_{g} .
$$

Clearly, $\mathbf{x}^{2}+p \mathbf{x}+q=0$. Let $\iota: K_{g} \rightarrow K_{g}$ be the only nontrivial $K$-linear automorphism (involution) of $K_{g}$. We write

$$
\operatorname{Tr}: K_{g} \rightarrow K, z \mapsto z+\iota(z), \text { Norm }: K_{g} \rightarrow K, z \mapsto z \cdot \iota(z)
$$

for the trace and norm maps attached to $K_{g} / K$. We have

$$
z^{2}-\operatorname{Tr}(z)+\operatorname{Norm}(z)=(z-z)(z-\iota(z))=0 \forall z \in K_{g} .
$$

For each $a \in K$ we have

$$
g(a)=\operatorname{Norm}(a-\mathbf{x})
$$

We also have

$$
\iota(\mathbf{x})=-p-\mathbf{x}, \operatorname{Norm}(\mathbf{x})=q, \operatorname{Tr}(\mathbf{x})=-p .
$$

In addition, we have the following equality in $K_{g}[x]$ :

$$
g(x)=x^{2}+p x+q=(x-\mathbf{x})(x-\iota \mathbf{x})=(x-\mathbf{x})(x+p+\mathbf{x}) .
$$

Let us consider the elliptic curve

$$
E=\mathcal{E}_{\alpha, p, q}: y^{2}=f(x):=(x-\alpha) g(x) .
$$

Clearly, $E(K)$ contains exactly one point of order 2 , namely, $(\alpha, 0)$. On the other hand, $E\left(K_{g}\right)$ contains two remaining points of order 2 , namely, $(\mathbf{x}, 0)$ and $(\iota \mathbf{x}, 0)=$ $(-p-\mathbf{x}, 0)$.

We start with the following result that was inspired by a paper of Schaefer [6].

Theorem 3.1. A point $P=\left(x_{0}, y_{0}\right) \in E(K)$ lies in $2 E(K)$ if and only if $x_{0}-\mathbf{x}$ is a square in $K_{g}$. If this is the case, then $x_{0}-\alpha$ is a square in $K$.

Remark 3.2. $\quad$ (i) If $x_{0} \in K$, then $x_{0}-\mathbf{x} \neq 0$, because $\mathbf{x} \notin K$. 
(ii) Suppose that $\mathbf{r} \in K_{g}$ and $r \in K$ satisfy

$$
r^{2}=x_{0}-\alpha, \mathbf{r}^{2}=x_{0}-\mathbf{x} .
$$

Then

$$
(r \cdot \operatorname{Norm}(\mathbf{r}))^{2}=r^{2} \cdot \operatorname{Norm}\left(x_{0}-\mathbf{x}\right)=\left(x_{0}-\alpha\right) g\left(x_{0}\right)=y_{0}^{2} .
$$

This means that

In addition,

$$
\begin{gathered}
(\operatorname{Tr}(\mathbf{r}))^{2}=(\mathbf{r}+\iota(\mathbf{r}))^{2}=\mathbf{r}^{2}+2 \mathbf{r} \iota(\mathbf{r})+\iota(\mathbf{r})^{2}=\left(\mathbf{r}^{2}+\iota\left(\mathbf{r}^{2}\right)\right)+2 \operatorname{Norm}(\mathbf{r}) \\
=\operatorname{Tr}\left(x_{0}-\mathbf{x}\right)+2 \operatorname{Norm}(\mathbf{r})=2 x_{0}+p+2 \operatorname{Norm}(\mathbf{r}) .
\end{gathered}
$$

It follows that

$$
\begin{aligned}
& \text { Notice also that } \\
& \qquad \begin{array}{l}
\operatorname{Norm}(r+\mathbf{r})=(r+\mathbf{r}) \iota(r+\mathbf{r})=(r+\mathbf{r})(r+\iota(\mathbf{r})) \\
=r^{2}+r(\mathbf{r}+\iota(\mathbf{r}))+\mathbf{r} \iota(\mathbf{r})=\left(x_{0}-\alpha\right)+r \operatorname{Tr}(\mathbf{r})+\operatorname{Norm}(\mathbf{r}) .
\end{array}
\end{aligned}
$$$$
(\operatorname{Tr}(\mathbf{r}))^{2}=2 x_{0}+p+2 \operatorname{Norm}(\mathbf{r}) .
$$

Consequently,

$$
\operatorname{Norm}(r+\mathbf{r})=\left(x_{0}-\alpha\right)+r \operatorname{Tr}(\mathbf{r})+\operatorname{Norm}(\mathbf{r}) .
$$

The next assertion describes explicitly both $\frac{1}{2} P$ 's in $E(K)$.

Theorem 3.3. Suppose that $P=\left(x_{0}, y_{0}\right) \in E(K)$. Suppose that $r \in K$ and $\mathbf{r} \in K_{g}$ satisfy

$$
r^{2}=x_{0}-\alpha, \mathbf{r}^{2}=x_{0}-\mathbf{x}, r \operatorname{Norm}(\mathbf{r})=-y_{0} .
$$

Then:

(i) the points

$$
\begin{aligned}
& Q_{r, \mathbf{r}}:=Q_{\mathbf{r}, \iota(\mathbf{r}), r}\left(x_{1}, y_{1}\right):=\left(x_{0}+\operatorname{Tr}(\mathbf{r}) r+\operatorname{Norm}(\mathbf{r}),-y_{0}-(r+\operatorname{Tr}(\mathbf{r}))(\operatorname{Tr}(\mathbf{r}) r+\operatorname{Norm}(\mathbf{r}))\right)= \\
& \begin{array}{r}
\text { and } \quad \alpha+\operatorname{Norm}(r+\mathbf{r}),-\operatorname{Tr}(\mathbf{r}) \operatorname{Norm}(r+\mathbf{r})) \\
Q_{r,-\mathbf{r}}:=Q_{-\mathbf{r},-\iota(\mathbf{r}), r}=\left(x_{-1}, y_{-1}\right):= \\
\left(x_{0}-\operatorname{Tr}(\mathbf{r}) r+\operatorname{Norm}(\mathbf{r}),-y_{0}-(r-\operatorname{Tr}(\mathbf{r}))(-\operatorname{Tr}(\mathbf{r}) r+\operatorname{Norm}(\mathbf{r}))\right) \\
=(\alpha+\operatorname{Norm}(r-\mathbf{r}), \operatorname{Tr}(\mathbf{r}) \operatorname{Norm}(r-\mathbf{r}))
\end{array}
\end{aligned}
$$

are distinct points of $E(K)$ that satisfy

$$
2 Q_{r, \mathbf{r}}=2 Q_{r,-\mathbf{r}}=P \in E(K) .
$$

(ii) The tangent lines to $E$ at $Q_{r, \mathbf{r}}$ and $Q_{r,-\mathbf{r}}$ are defined by the equations

$$
\mathcal{L}_{r, \mathbf{r}}: y=l_{r, \mathbf{r}}\left(x-x_{0}\right)-y_{0} \text { and } \mathcal{L}_{r,-\mathbf{r}}: y=l_{r,-\mathbf{r}}\left(x-x_{0}\right)-y_{0},
$$

respectively, where

$$
l_{r, \mathbf{r}}=-(r+\operatorname{Tr}(\mathbf{r})), l_{r,-\mathbf{r}}=-(r-\operatorname{Tr}(\mathbf{r})) .
$$

Remark 3.4. Suppose we are given $\mathbf{r} \in K_{g}$ with $\mathbf{r}^{2}=x_{0}-\mathbf{x}$. It follows from Theorem 3.1 and (12) that there exists precisely one $r \in K$ such that $r^{2}=x_{0}-\alpha$ and $r \operatorname{Norm}(\mathbf{r})=-y_{0}$. 
Proof of Theorem 3.1 and 3.3. We start with the proof of Theorem 3.1. Suppose that $P=\left(x_{0}, y_{0}\right) \in 2 E(K)$. Since $E\left(K_{g}\right)$ contains all three points of order 2 on $E$ and $(\mathbf{x}, 0)$ is one of them; it follows from Theorem 2.3 that $x_{0}-\mathbf{x}$ is a square in $K_{g}$.

Conversely, suppose that $x_{0}-\mathbf{x}$ is a square in $K_{g}$. This means that there exists $\mathbf{r} \in K_{g}$ such that

$$
x_{0}-\mathbf{x}=\mathbf{r}^{2}=(-\mathbf{r})^{2} .
$$

By Remark 3.2(i), $\mathbf{r}$ does not lie in $K$. In particular, $\mathbf{r} \neq 0$. We have

$$
x_{0}-\iota(\mathbf{x})=\iota\left(x_{0}-\mathbf{x}\right)=\iota\left(\mathbf{r}^{2}\right)=\iota(\mathbf{r})^{2} .
$$

This implies that

$$
g\left(x_{0}\right)=\left(x_{0}-\mathbf{x}\right)\left(x_{0}-\iota \mathbf{x}\right)=(\mathbf{r} \iota(\mathbf{r}))^{2}=\operatorname{Norm}(\mathbf{r})^{2}
$$

is a square in $K$, because $\operatorname{Norm}(\mathbf{r}) \in K$. Since $\left(x_{0}, y_{0}\right) \in E(K)$,

$$
y_{0}^{2}=f\left(x_{0}\right)=\left(x_{0}-\alpha\right) g\left(x_{0}\right)=\left(x_{0}-\alpha\right) \operatorname{Norm}(\mathbf{r})^{2}
$$

is a square in $K$. This implies that $x_{0}-\alpha$ is also a square in $K$, because the norm of nonzero $\mathbf{r}$ also does not vanish. It follows that $x_{0}-\alpha$ is a square in $K_{g}$ as well. By Remark 3.2(ii), we may choose

$$
r=\sqrt{x_{0}-\alpha} \in K \subset K_{g}
$$

in such a way that

$$
r \operatorname{Norm}(\mathbf{r})=r \operatorname{Norm}(-\mathbf{r})=r \cdot \mathbf{r} \cdot \iota(\mathbf{r})=-y_{0} .
$$

Since all three $x_{0}-\mathbf{x}, x_{0}-\iota(\mathbf{x})$, and $x_{0}-\alpha$ are squares in $K_{g}$, Theorem 2.3 implies that

$$
P=\left(x_{0}, y_{0}\right) \in 2 E\left(K_{g}\right) ;
$$

by formula (6) combined with the condition $r \operatorname{Norm}(\mathbf{r})=-y_{0}$ and equality (14), the two different choices $(\mathbf{r}, \iota(\mathbf{r}), r)$ and $(-\mathbf{r},-\iota(\mathbf{r}), r)$ of the corresponding square roots give us

$$
\begin{gathered}
\left.Q_{r, \mathbf{r}}=\left(x_{0}+(\mathbf{r}+\iota(\mathbf{r})) r+\mathbf{r} \iota(\mathbf{r})\right),-y_{0}-(r+\mathbf{r}+\iota(\mathbf{r}))((\mathbf{r}+\iota(\mathbf{r})) r+\mathbf{r} \iota(\mathbf{r}))\right) \\
=\left(x_{0}+\operatorname{Tr}(\mathbf{r}) r+\operatorname{Norm}(\mathbf{r}),-y_{0}-(r+\operatorname{Tr}(\mathbf{r}))(\operatorname{Tr}(\mathbf{r}) r+\operatorname{Norm}(\mathbf{r}))\right) \\
=(\alpha+\operatorname{Norm}(r+\mathbf{r}),-\operatorname{Tr}(\mathbf{r}) \operatorname{Norm}(r+\mathbf{r}))
\end{gathered}
$$

and

$$
\begin{gathered}
Q_{r,-\mathbf{r}}=\left(x_{0}-(\mathbf{r}+\iota(\mathbf{r})) r+\mathbf{r} \iota(\mathbf{r}),-y_{0}-(r-\mathbf{r}-\iota(\mathbf{r}))((-\mathbf{r}-\iota(\mathbf{r})) r+\mathbf{r} \iota(\mathbf{r}))\right)= \\
(\alpha+\operatorname{Norm}(-r+\mathbf{r}), \operatorname{Tr}(\mathbf{r}) \operatorname{Norm}(r-\mathbf{r}))= \\
\left(x_{0}-\operatorname{Tr}(\mathbf{r}) r+\operatorname{Norm}(\mathbf{r}),-y_{0}-(r-\operatorname{Tr}(\mathbf{r}))(-\operatorname{Tr}(\mathbf{r}) r+\operatorname{Norm}(\mathbf{r}))\right)
\end{gathered}
$$

that are distinct points of $E\left(K_{g}\right)$ such that

$$
2 Q_{r, \mathbf{r}}=2 Q_{r,-\mathbf{r}}=P \in E(K) .
$$

However, it is clear that they both lie in $E(K)$, since all three $r, \operatorname{Tr}(\mathbf{r})$, and $\operatorname{Norm}(\mathbf{r})$ lie in $K$. This ends the proof of Theorem 3.1. On the other hand, $Q_{r, \mathbf{r}}$ and $Q_{r,-\mathbf{r}}$ are exactly the points that appear in the statement of Theorem 3.3. this proves Theorem 3.3(i) as well. Theorem 3.3(ii) follows from the explicit formula (7) for the slope $l$ of the tangent line at $\frac{1}{2} P$. 
Remark 3.5. If $P=\left(x_{0}, y_{0}\right) \in 2 E(K)$, then $g\left(x_{0}\right)$ is a square in $K$. Indeed, we have

$$
y_{0}^{2}=f\left(x_{0}\right)=g\left(x_{0}\right)\left(x_{0}-\alpha\right), g\left(x_{0}\right)=\operatorname{Norm}\left(x_{0}-\mathbf{x}\right) .
$$

Since $x_{0}-\mathbf{x}$ is a square in $K_{g}$, its norm $g\left(x_{0}\right)$ is a square in $K$.

Example 3.6. Suppose $g(x)=x^{2}+p x+q \in K[x]$ is an irreducible quadratic polynomial over $K$ and $-\mathbf{x}$ is a square in $K_{g}$, i.e., there are exist $u, v \in K$ such that

$$
-\mathbf{x}=(u \mathbf{x}+v)^{2}
$$

in $K_{g}$. This means that the polynomial $(u x+v)^{2}+x$ is divisible by $x^{2}+p x+q$ in $K[x]$, i.e., the polynomial

$$
\left(2 u v+1-u^{2} p\right) x+\left(v^{2}-u^{2} q\right)=(u x+v)^{2}+x-u^{2}\left(x^{2}+p x+q\right)
$$

of degree $<2$ is divisible by quadratic $x^{2}+p x+q$ in $K[x]$ and therefore is identically zero, i.e.,

$$
2 u v+1=u^{2} \cdot p, v^{2}=u^{2} q .
$$

In addition, $u \neq 0$, since $-\mathbf{x} \in K_{g} \backslash K$. This implies that there exists $b \in K$ such that

It follows that

$$
q=b^{2}, v=u b .
$$

$$
u^{2} \cdot p=2 u^{2} b+1,(p-2 b) u^{2}=1 \text {. }
$$

This means that

$$
p=2 b+\frac{1}{u^{2}}, q=b^{2}, v=u b .
$$

Let us put

Then we obtain that

$$
a:=\frac{1}{u} \in K \backslash\{0\}
$$

$$
\begin{gathered}
p=a^{2}+2 b, q=b^{2}, \\
g(x)=x^{2}+\left(a^{2}+2 b\right) x+b^{2} ; u=\frac{1}{a}, v=\frac{b}{a},
\end{gathered}
$$

and

$$
\left(\frac{1}{a} \mathbf{x}+\frac{b}{a}\right)^{2}=-\mathbf{x}
$$

in $K_{g}$. Since $g(x)$ is irreducible over $K$, its discriminant

$$
\left(a^{2}+2 b\right)^{2}-4 b^{2}=a^{4}+4 a^{2} b=a^{2}\left(a^{2}+4 b\right)
$$

is not a square in $K$, i.e, $a^{2}+4 b$ is not a square in $K$. In addition, the constant term $b^{2}$ of $g(x)$ does not vanish, i.e., $b \neq 0$. Now let us consider the $K$-point $W=(0,0)$ of the elliptic curve

$$
\mathcal{E}_{a, b}^{(4)}=\mathcal{E}_{0, a^{2}+2 b, b^{2}}: y^{2}=\left(x^{2}+\left(a^{2}+2 b\right) x+b^{2}\right) x .
$$

Clearly, $W$ has order 2. It follows from Theorem 3.1 that $W$ is divisible by 2 in $E(K)$, i.e., there are two points $Q_{+}$and $Q_{-}$of order 4 in $\mathcal{E}_{a, b}^{(4)}(K)$ such that

$$
2 Q_{+}=2 Q_{-}=W \text {. }
$$

In order to find explicitly $Q_{+}$and $Q_{-}$, let us apply Theorem 3.3 . We have

$$
r=0, \mathbf{r}=\frac{1}{a}(\mathbf{x}+b)
$$


FAMILIES OF ELLIPTIC CURVES WITH RATIONAL TORSION POINTS OF EVEN ORDER 9

$$
\begin{gathered}
\operatorname{Tr}(\mathbf{r})=\frac{1}{a}(-p)+2 \cdot \frac{b}{a}=-\frac{\left(a^{2}+2 b\right)}{a}+\frac{2 b}{a}=-a, \\
\operatorname{Norm}(\mathbf{r})=\frac{\operatorname{Norm}(\mathbf{x}+b)}{a^{2}}=\frac{g(-b)}{a^{2}}=-b .
\end{gathered}
$$

Now the explicit formulas of Theorem 3.3 give us

$$
Q_{+}=(-b, a b), Q_{+}=(-b,-a b) .
$$

\section{ANOther CRITERION OF DIVISIBILITY By 2}

We keep the notation of Section 3 However, we drop the assumption that $g(x)=x^{2}+p x+q \in K[x]$ is irreducible over $K$ and assume only that it has no multiple roots. Let $\alpha_{1}, \alpha_{2}$ be (distinct) roots of $g(x)$ in $\bar{K}$. We have

$$
\alpha_{1}+\alpha_{2}=-p, \alpha_{1} \alpha_{2}=q \text {. }
$$

Theorem 4.1. Let $\alpha$ be an element of $K$ that is not a root of $g(x)$. Let us consider the elliptic curve

over K. Let

$$
E: y^{2}=(x-\alpha) g(x)
$$

$$
W_{3}=\left(\alpha_{3}, 0\right)=(\alpha, 0) \in E(K)
$$

be a point of order 2 .

Let $P=\left(x_{0}, y_{0}\right)$ be a $K$-point of $E$. Then $P$ is divisible by 2 in $E(K)$ if and only if the following two conditions hold.

(i) The difference $x_{0}-\alpha$ is a square in $K$.

(ii) There exist square roots $r=\sqrt{x_{0}-\alpha} \in K$ and

$$
r_{i}=\sqrt{x_{0}-\alpha_{i}} \in \bar{K}(i=1,2)
$$

such that

$$
r_{1} r_{2} r=-y_{0} ; r_{1}+r_{2} \in K .
$$

In addition, if square roots $r_{1}, r_{2}, r_{3}$ satisfy conditions (ii), then

$$
Q_{+}=Q_{r_{1}, r_{2}, r}=\left(x_{0}+\left(r_{1}+r_{2}\right) r+r_{1} r_{2},-y_{0}-\left(\left(r_{1}+r_{2}\right)+r\right)\left(\left(r_{1}+r_{2}\right) r+r_{1} r_{2}\right)\right.
$$

and

$Q_{-}=Q_{-r_{1},-r_{2}, r}=\left(x_{0}-\left(r_{1}+r_{2}\right) r+r_{1} r_{2},-y_{0}-\left(-\left(r_{1}+r_{2}\right)+r\right)\left(-\left(r_{1}+r_{2}\right) r+r_{1} r_{2}\right)\right.$

are distinct points of $E(K)$, which satisfy

$$
2 Q_{+}=2 Q_{-}=P, Q_{-}=Q_{+}+W_{3} .
$$

Conversely, if $Q \in E(K)$ satisfies $2 Q=P$, then there exist square roots $r, r_{1}, r_{2}$ that satisfy conditions (ii) and $Q=Q_{r_{1}, r_{2}, r}$.

Remark 4.2. If $x_{0} \neq \alpha$, i.e., $P \neq(\alpha, 0)$, then $r=\sqrt{x_{0}-\alpha} \neq 0$ and

$$
r_{1} r_{2}=-\frac{y_{0}}{r} \text {. }
$$

It follows from Theorem 4.1 that

$$
P=\left(x_{0}, y_{0}\right) \in E(K)
$$

is divisible by 2 in $E(K)$ if and only if there exist square roots

$$
r=\sqrt{x_{0}-\alpha} \in K, \quad r_{i}=\sqrt{x_{0}-\alpha_{i}} \in \bar{K}(i=1,2)
$$


such that

$$
r_{1} r_{2}=-\frac{y_{0}}{r}, \quad r_{1}+r_{2} \in K .
$$

Proof of Theorem 4.1. Let us put

$$
\alpha_{3}=\alpha, W_{3}=\left(\alpha_{3}, 0\right) \in E(K) .
$$

Suppose that conditions (i)-(ii) hold. Let us put

$$
r_{3}=r \in K .
$$

We have

$$
\begin{array}{r}
\left(r_{1}+r_{2}\right)^{2}=r_{1}^{2}+2 r_{1} r_{2}+r_{2}^{2}=\left(x_{0}-\alpha_{1}\right)+2 r_{1} r_{2}+\left(x_{0}-\alpha_{2}\right) \\
=2 x_{0}-\left(\alpha_{1}+\alpha_{2}\right)+2 r_{1} r_{2}=\left(2 x_{0}+p\right)+2 r_{1} r_{2} .
\end{array}
$$

Since all three $r_{1}+r_{2}, x_{0}, p$ lie in $K$, we obtain that

$$
r_{1} r_{2} \in K \text {. }
$$

Notice that

(18) $s_{1}=r_{1}+r_{2}+r_{3}=\left(r_{1}+r_{2}\right)+r, s_{2}=r_{1} r_{2}+r_{2} r_{3}+r_{3} r_{1}=\left(r_{1}+r_{2}\right) r+r_{1} r_{2}$.

Clearly, both $s_{1}$ and $s_{2}$ lie in $K$. Let us put

$$
\begin{gathered}
x_{1}=x_{0}+r_{1} r_{2}+r_{2} r_{3}+r_{3} r_{1}=x_{0}+s_{2}, \\
y_{1}=-y_{0}-\left(r_{1}+r_{2}+r_{3}\right)\left(r_{1} r_{2}+r_{2} r_{3}+r_{3} r_{1}\right)=-y_{0}-s_{1} s_{2} .
\end{gathered}
$$

Since all $x_{0}, y_{0}, s_{1}, s_{2}$ lie in $K$, both $x_{1}$ and $y_{1}$ also lie in $K$. By formula (6), the point $Q_{+}=Q_{r_{1}, r_{2}, r_{3}}=\left(x_{1}, y_{1}\right)$ lies in $E(\bar{K})$ and satisfies $2 Q_{+}=P$. Since $x_{1}$ and $y_{1}$ lie in $K$, the point $Q_{+}$actually lies in $E(K)$, and therefore $P$ is divisible by 2 in $E(K)$. Similarly, the triple of square roots $\left(-r_{1},-r_{2}, r_{3}\right)$ satisfies (11) and (4); in addition,

$$
\left(-r_{1}\right)+\left(-r_{2}\right)=-\left(r_{1}+r_{2}\right) \in K,\left(-r_{1}\right)\left(-r_{2}\right)-r_{1} r_{2} .
$$

This implies that

$$
Q_{-}=Q_{-r_{1},-r_{2}, r_{3}}=Q_{r_{1}, r_{2}, r_{3}}+W_{3}=Q_{+}+W_{3}
$$

also lies in $E(K)$ and satisfies $2 Q_{-}=P$.

Conversely, suppose that $P=2 Q$ with $Q=\left(x_{1}, y_{1}\right) \in E(K)$. We claim that $x_{0}-\alpha_{3}=x_{0}-\alpha$ is a square in $K$. Indeed, if $g(x)$ is irreducible over $K$, then our claim follows from Theorem 3.1. If $g(x)$ is reducible, i.e., $\alpha_{1}, \alpha_{2}$ and $\alpha_{3}$ lie in $K$, then our claim follows from Theorem 2.3

It follows from Theorem 2.5 that there exist square roots $r_{i}=\sqrt{x_{0}-\alpha_{i}}$ such that

$$
r_{1} r_{2} r_{3}=-y_{0}, r_{1}+r_{2}+r_{3} \in K, r_{1} r_{2}+r_{2} r_{1}+r_{3} r_{1} \in K
$$

and

Notice that

$$
Q=\left(x_{1}, y_{1}\right)=Q_{r_{1}, r_{2}, r_{3}}
$$

$$
r:=r_{3}=\sqrt{x_{0}-\alpha_{3}}=\sqrt{x_{0}-\alpha} \in K
$$

and therefore

$$
r_{1} r_{2} r=r_{1} r_{2} r_{3}=-y_{0}, Q=Q_{r_{1}, r_{2}, r_{3}}=Q_{r_{1}, r_{2}, r} .
$$

Since $r_{3}=r \in K$, we conclude that $r_{1}+r_{2}=\left(r_{1}+r_{2}+r_{3}\right)-r_{3} \in K$. 
FAMILIES OF ELLIPTIC CURVES WITH RATIONAL TORSION POINTS OF EVEN ORDER1

Remark 4.3. Let $Q=\left(x_{1}, y_{1}\right) \in E(K)$ with $y_{1} \neq 0$ (i.e., $Q$ is none of $W_{j}$ ) and $P=2 Q \in E(K)$. Then $Q=Q_{r_{1}, r_{2}, r_{3}}$ for suitable $r_{k}=\sqrt{x(P)-\alpha_{k}}$, and formulas (9) (with $i=3$ ) tell us that

$$
\begin{array}{r}
r_{3}=-\frac{y_{1}}{2} \cdot( \\
\left.-\frac{1}{x_{1}-\alpha}+\frac{1}{x_{1}-\alpha_{1}}+\frac{1}{x_{1}-\alpha_{2}}\right) \\
=-\frac{y_{1}}{2} \cdot\left(-\frac{1}{x_{1}-\alpha}+\frac{2 x_{1}+p}{g\left(x_{1}\right)}\right) .
\end{array}
$$

In particular, if $x_{1}=0$ and $\alpha=-1$, then $y_{1}^{2}=q, g\left(x_{1}\right)=q$ and

$$
r_{3}^{2}=\frac{q}{4} \cdot\left(-1+\frac{p}{q}\right)^{2}=\frac{(p-q)^{2}}{4 q}
$$

and

$$
x(P)=x(2 Q)=\alpha+r_{3}^{2}=\frac{(p-q)^{2}}{4 q}-1 .
$$

Theorem 4.4. We keep the notation and assumptions of Theorem 4.1. Assume additionally that $x_{0} \neq \alpha$, i.e., $P \neq(\alpha, 0)$. Then $P=\left(x_{0}, y_{0}\right)$ is divisible by 2 in $E(K)$ if and only if there exists $r \in K$ such that $r^{2}=x_{0}-\alpha$ and $\left(2 x_{0}+p\right)\left(x_{0}-\right.$ $\alpha)-2 y_{0} r$ is a square in $K$.

In addition, if these equivalent conditions hold and we choose

$$
T=\frac{\sqrt{\left(2 x_{0}+p\right)\left(x_{0}-\alpha\right)-2 y_{0} r}}{r} \in K,
$$

then

$$
\mathcal{Q}_{r, T}=\left(x_{1}, y_{1}\right):=\left(x_{0}+r T-\frac{y_{0}}{r},-y_{0}-(r+T)\left(r T+\frac{y_{0}}{r}\right)\right)
$$

and

$$
\mathcal{Q}_{r,-T}=\left(x_{-1}, y_{-1}\right):=\left(x_{0}-r T-\frac{y_{0}}{r},-y_{0}-(r-T)\left(-r T+\frac{y_{0}}{r}\right)\right)
$$

are distinct points of $E(K)$, which satisfy

$$
2 \mathcal{Q}_{r, T}=2 \mathcal{Q}_{r,-T}=P \in E(K), \mathcal{Q}_{r,-T}=\mathcal{Q}_{r, T}+W_{3} .
$$

Conversely, if $Q \in E(Q)$ satisfies $2 Q=P$, then there exist

$$
r=\sqrt{x_{0}-\alpha} \in K \quad \text { and } T=\frac{\sqrt{\left(2 x_{0}+p\right)\left(x_{0}-\alpha\right)-2 y_{0} r}}{r} \in K
$$

such that $Q=\mathcal{Q}_{r, T}$.

Proof. In light of Remark 4.2, the divisibility of $P$ by 2 in $E(K)$ is equivalent to the existence of square roots $r=\sqrt{x_{0}-\alpha} \in K$ and $r_{i}=\sqrt{x_{0}-\alpha_{i}} \in \bar{K}(i=1,2)$ such that

$$
r_{1} r_{2}=-\frac{y_{0}}{r}, r_{1}+r_{2} \in K .
$$

Suppose such $r_{1}, r_{2}, r$ exist. Then $\left(r_{1}+r_{2}\right)^{2}$ is a square in $K$. We have

$$
\begin{array}{r}
\left(r_{1}+r_{2}\right)^{2}=r_{1}^{2}+2 r_{1} r_{2}+r_{2}^{2}=\left(x_{0}-\alpha_{1}\right)-2 \frac{y_{0}}{r}+\left(x_{0}-\alpha_{2}\right) \\
=2 x_{0}+p-2 \frac{y_{0}}{r}=\frac{\left(2 x_{0}+p\right)\left(x_{0}-\alpha\right)-2 y_{0} r}{r^{2}} .
\end{array}
$$

This implies that $\left(2 x_{0}+p\right)\left(x_{0}-\alpha\right)-2 y_{0} r$ is a square in $K$. 
Conversely, suppose that there exists $r \in K$ such that $r^{2}=x_{0}-\alpha$ and $\left(2 x_{0}+\right.$ $p)\left(x_{0}-\alpha\right)-2 y_{0} r$ is a square in $K$. This implies that

$$
\frac{\left(2 x_{0}+p\right)\left(x_{0}-\alpha\right)-2 y_{0} r}{r^{2}}=\frac{\left(2 x_{0}+p\right)\left(x_{0}-\alpha\right)-2 y_{0} r}{x_{0}-\alpha}=2 x_{0}+p-\frac{2 y_{0}}{r}
$$

is a square in $K$. Let us put $r_{3}=r$ and choose square roots $r_{i}=\sqrt{x_{0}-\alpha_{i}} \in \bar{K}$ $(i=1,2)$ in such a way that $r_{1} r_{2} r_{3}=-y_{0}$. (Since $r_{3}=r \neq 0$, the only other choice of such a pair of square roots is $\left(-r_{1},-r_{2}\right)$.) Let

$$
T:=r_{1}+r_{2} \text {. }
$$

We have

$$
r_{1} r_{2}=-\frac{y_{0}}{r} \in K
$$

and

$$
\begin{gathered}
T^{2}=\left(r_{1}+r_{2}\right)^{2}=r_{1}^{2}+2 r_{1} r_{2}+r_{2}^{2}=\left(x_{0}-\alpha_{1}\right)-2 \frac{y_{0}}{r}+\left(x_{0}-\alpha_{2}\right)= \\
2 x_{0}+p-2 \frac{y_{0}}{r}=\frac{\left(2 x_{0}+p\right)\left(x_{0}-\alpha\right)-2 y_{0} r}{r^{2}}
\end{gathered}
$$

is a square in $K$. This implies that

$$
T=r_{1}+r_{2} \in K \text {. }
$$

It follows from Remark 4.2 that $P$ is divisible by 2 in $E(K)$. By Theorem 4.1 we obtain that

$$
Q_{+}=Q_{r_{1}, r_{2}, r}=\left(x_{0}+\left(r_{1}+r_{2}\right) r+r_{1} r_{2},-y_{0}-\left(\left(r_{1}+r_{2}\right)+r\right)\left(\left(r_{1}+r_{2}\right) r+r_{1} r_{2}\right)\right.
$$

and

$Q_{-}=Q_{-r_{1},-r_{2}, r}=\left(x_{0}-\left(r_{1}+r_{2}\right) r+r_{1} r_{2},-y_{0}-\left(-\left(r_{1}+r_{2}\right)+r\right)\left(-\left(r_{1}+r_{2}\right) r+r_{1} r_{2}\right)\right.$ are distinct points of $E(K)$, which satisfy

$$
2 Q_{+}=2 Q_{-}=P, Q_{-}=Q_{+}+W_{3} .
$$

Taking into account that

$$
r_{1}+r_{2}=T, r_{1} r_{2}=-\frac{y_{0}}{r}
$$

we obtain that

$$
\mathcal{Q}_{r, T}=Q_{+}, \mathcal{Q}_{r,-T}=Q_{-},
$$

and therefore

$$
\begin{gathered}
\mathcal{Q}_{r, T}, \mathcal{Q}_{r,-T} \in E(K), \mathcal{Q}_{r,-T}=\mathcal{Q}_{r, T}+W_{3}, \\
2 \mathcal{Q}_{r, T}=2 \mathcal{Q}_{r,-T}=P .
\end{gathered}
$$

On the other hand, if $P=2 Q$ with $Q \in E(K)$, then it follows from Theorem 4.1 that there exist square roots $r=\sqrt{x_{0}-\alpha} \in K$ and

$$
r_{i}=\sqrt{x_{0}-\alpha_{i}} \in \bar{K}(i=1,2)
$$

such that

$$
r_{1} r_{2} r=-y_{0}, r_{1}+r_{2} \in K
$$

and $Q=Q_{r_{1}, r_{2}, r}$. This implies that $r_{1} r_{2}=-y_{0} / r$. Now if we put $T=\left(r_{1}+r_{2}\right) \in K$, then (as we have already seen)

$$
T^{2}=\left(r_{1}+r_{2}\right)^{2}=\left(2 x_{0}+p\right)\left(x_{0}-\alpha\right)-2 y_{0} r \in K .
$$


FAMILIES OF ELLIPTIC CURVES WITH RATIONAL TORSION POINTS OF EVEN ORDER3

Now one can easily check that

$Q_{r_{1}, r_{2}, r}=\left(x_{0}+\left(r_{1}+r_{2}\right) r+r_{1} r_{2},-y_{0}-\left(\left(r_{1}+r_{2}\right)+r\right)\left(\left(r_{1}+r_{2}\right) r+r_{1} r_{2}\right)=\mathcal{Q}_{r, T}\right.$, and therefore $Q=\mathcal{Q}_{r, T}$.

Remark 4.5. It is known (3) that $r_{1}+r_{2} \neq 0$. In light of (21),

$$
\left(2 x_{0}+p\right)\left(x_{0}-\alpha\right)-2 y_{0} r \neq 0 .
$$

Theorem 4.6. Let $\alpha$ be an element of $K$ that is not a root of $g(x)$. Let us consider the elliptic curve

$$
E: y^{2}=g(x)(x-\alpha)
$$

over $K$. Let $y_{0}$ be a nonzero element of $K$. Then

$$
P=\left(0, y_{0}\right) \in 2 E(K) \subset E(K)
$$

if and only if there exists $r \in K$ and a nonzero $T \in K$ such that

$$
r^{2}=-\alpha, g(x)=x^{2}+\left(T^{2}+2 \frac{y_{0}}{r}\right) x+\left(\frac{y_{0}}{r}\right)^{2} .
$$

If (22) holds, then the equation for $E$ becomes

$$
y^{2}=\left(x+r^{2}\right)\left(x^{2}+\left(T^{2}+2 \frac{y_{0}}{r}\right) x+\left(\frac{y_{0}}{r}\right)^{2}\right) .
$$

In addition,

and

$$
\mathcal{Q}_{r, T}=\left(r T-\frac{y_{0}}{r},-y_{0}-(r+T)\left(r T-\frac{y_{0}}{r}\right)\right)
$$

$$
\mathcal{Q}_{r,-T}=\left(-r T-\frac{y_{0}}{r},-y_{0}-(r-T)\left(-r T-\frac{y_{0}}{r}\right)\right)
$$

are distinct points of $E(K)$ that satisfy

$$
2 \mathcal{Q}_{r, T}=2 \mathcal{Q}_{r,-T}=P, \mathcal{Q}_{r,-T}=\mathcal{Q}_{r, T}+W_{3} .
$$

Moreover, if $Q \in E(K)$ satisfies $2 Q=P$, then there exist

$$
r \in K \text { and } T \in K
$$

that enjoy property (22) and $Q=\mathcal{Q}_{r, T}$.

Proof. We have the equation for $E$

$$
y^{2}=\left(x^{2}+p x+q\right)(x-\alpha) .
$$

Since $P=\left(x_{0}, y_{0}\right)=\left(0, y_{0}\right) \in E(K)$, we have $y_{0}^{2}=q \cdot(-\alpha)$, which means that

$$
q=\frac{y_{0}^{2}}{-\alpha} \text {. }
$$

In light of Theorem 4.4 the inclusion $P \in 2 E(K)$ is equivalent to the existence of nonzero $r, T \in K$ such that

$$
r^{2}=-\alpha, T^{2}=\frac{\left(2 x_{0}+p\right)\left(x_{0}-\alpha\right)-2 y_{0} r}{r^{2}}=\frac{p r^{2}-2 y_{0} r}{r^{2}}=p-2 \frac{y_{0}}{r} .
$$

This means that

i.e.,

$$
q=\left(\frac{y_{0}}{r}\right)^{2}, p=T^{2}+2 \frac{y_{0}}{r}
$$

$$
g(x)=x^{2}+p x+q=x^{2}+\left(T^{2}+2 \frac{y_{0}}{r}\right) x+\left(\frac{y_{0}}{r}\right)^{2} .
$$


The assertions about $\mathcal{Q}_{r, T}, \mathcal{Q}_{r,-T}$, and $Q=\frac{1}{2} P$ follow from the corresponding assertions of Theorem 4.4 .

Remark 4.7. Dividing both sides of (23) by $r^{6}$ and making the substitution

$$
\tilde{x}=\frac{x}{r^{2}}, \tilde{y}=\frac{y}{r^{3}}, t=\frac{T}{r}, \tilde{y}_{0}=\frac{y_{0}}{r^{3}},
$$

we obtain that $E$ is isomorphic to the elliptic curve

$$
\tilde{E}_{t, \tilde{y}_{0}}: \tilde{y}^{2}=(\tilde{x}+1)\left(\tilde{x}^{2}+\left(t^{2}+2 \tilde{y}_{0}\right) \tilde{x}+\tilde{y}_{0}^{2}\right) \text {. }
$$

In addition, the isomorphism

$$
E \cong \tilde{E}_{t, \tilde{y}_{0}}:(x, y) \mapsto(\tilde{x}, \tilde{y})=\left(\frac{x}{r^{2}}, \frac{y}{r^{3}}\right)
$$

sends $W_{3}=\left(-r^{2}, 0\right) \in E(K)$ to $\tilde{W}_{3}=(-1,0) \in \tilde{E}_{t, \tilde{y}_{0}}(K), P$ to $\tilde{P}=\left(0, \tilde{y}_{0}\right) \in$ $\tilde{E}_{t, \tilde{y}_{0}}(K), \mathcal{Q}_{r, T}$ to $\mathcal{Q}_{1, t}=\left(t-\tilde{y}_{0},-\tilde{y}_{0}-(1+t)\left(t-\tilde{y}_{0}\right)\right)$, and $\mathcal{Q}_{r,-T}$ to $\mathcal{Q}_{1,-t}=$ $\left(-t-\tilde{y}_{0},-\tilde{y}_{0}-(1-t)\left(-t-\tilde{y}_{0}\right)\right)$. We also have

$$
2 \mathcal{Q}_{1, t}=2 \mathcal{Q}_{1,-t}=\tilde{P}, \mathcal{Q}_{1,-t}=\mathcal{Q}_{1, t}+\tilde{W}_{3} .
$$

\section{Elliptic CURVES With POINTS OF ORDER 4}

Let $E$ be an elliptic curve over a field $K$ with $\operatorname{char}(K) \neq 2$. Suppose that $E(K)$ contains exactly one point of order 2 . Then $E$ is $K$-isomorphic to

$$
\mathcal{E}_{0, p, q}: y^{2}=x\left(x^{2}+p x+q\right),
$$

where $x^{2}+p x+q \in K[x]$ is an irreducible quadratic polynomial.

Theorem 5.1. The following conditions are equivalent.

(i) $E(K)$ contains a cyclic subgroup of order 4.

(ii) There exist nonzero $a, b \in K$ such that $a^{2}+4 b$ is not a square in $K$ and $E$ is $K$-isomorphic to the elliptic curve

$$
\mathcal{E}_{a, b}^{(4)}: y^{2}=x\left(x^{2}+\left(a^{2}+2 b\right) x+b^{2}\right) .
$$

Proof. Suppose (i) holds. We may assume that $E=\mathcal{E}_{0, p, q}$. It follows from Example 3.6 that there are nonzero $a, b \in K$ such that $a^{2}+4 b$ is not a square in $K, p=a^{2}+2 b$, and $q=b^{2}$. This means that $E=\mathcal{E}_{a, b}^{(4)}$, i.e., (ii) holds.

Suppose (ii) holds. We may assume that

$$
E=\mathcal{E}_{a, b}^{(4)}: y^{2}=x\left(x^{2}+\left(a^{2}+2 b\right) x+b^{2}\right)
$$

with nonzero $a, b \in K$ and $a^{2}+4 b$ is not a square in $K$. (In particular, $a^{2}+4 b \neq 0$.) Clearly, $W=(0,0)$ is a point of order 2 in $E(K)$ and $P=(-b, a b) \in E(K)$. If we put $g(x)=x^{2}+\left(a^{2}+2 b\right) x+b^{2}$, then arguments of Example 3.6 show that $W$ is divisible by 2 in $\mathcal{E}_{a, b}^{(4)}(K)$, and therefore $\mathcal{E}_{a, b}^{(4)}(K)$ contains a point of order 4 . In fact, it contains exactly two points of order 4 , namely, $(-b, a b)$ and $(-b,-a b)$.

Remark 5.2. D. Kubert 5 described another family of elliptic curves

$$
\mathcal{E}_{4, t}: y^{2}+x y-t y=x^{3}-t x^{2}
$$

with point $Q=(0,0)$ of order 4 . The equation for $\mathcal{E}_{4, t}$ is equivalent to

$$
y^{2}+2 y \frac{(x-t)}{2}+\frac{(x-t)^{2}}{4}=(x-t) x^{2}+\frac{(x-t)^{2}}{4} \text {. }
$$


FAMILIES OF ELLIPTIC CURVES WITH RATIONAL TORSION POINTS OF EVEN ORDERI5

Now the change of variables

$$
\tilde{x}=x-t, \tilde{y}=y+\frac{(x-t)}{2}
$$

allows us to rewrite the equation as follows:

$$
\tilde{y}^{2}=\tilde{x}\left((\tilde{x}+t)^{2}+\frac{\tilde{x}}{4}\right)=\tilde{x}\left(\tilde{x}^{2}+\left(2 t+\frac{1}{4}\right) \tilde{x}+t^{2}\right) .
$$

This implies that $\mathcal{E}_{4, t}$ is isomorphic to $\mathcal{E}_{a, b}$ with $b=t, a=1 / 2$.

Theorem 5.3. Let $K$ be a field with $\operatorname{char}(K) \neq 2$, and let $a, b$ be nonzero elements of $K$ such that $a^{2}+4 b$ is not a square in $K$. (In particular, $a^{2}+4 b \neq 0$.) Let $c, d$ be nonzero elements of $K$ such that $c^{2}+4 d \neq 0$. Then the elliptic curves $\mathcal{E}_{a, b}$ and $\mathcal{E}_{c, d}$ are $K$-isomorphic if and only if there exists a nonzero $u \in K$ such that

$$
c^{2}+2 d=u^{2}\left(a^{2}+2 b\right), u^{4} d^{2}=b^{2} .
$$

Proof. Since $a^{2}+4 b$ is not a square in $K$, the quadratic polynomial $x^{2}+\left(a^{2}+2 b\right) x+b^{2}$ has no roots in $K$. This implies that $\mathcal{E}_{a, b}$ has exactly one point of order 2 , namely, $W_{a, b}=(0,0)$. Let $\phi: \mathcal{E}_{a, b} \cong \mathcal{E}_{c, d}$ be a $K$-isomorphism. Since $W_{c, d}=(0,0)$ is a point of order 2 in $\mathcal{E}_{c, d}(K)$, it is the only point of order 2 in $\mathcal{E}_{c, d}(K)$ and $\phi$ sends $W_{a, b}=(0,0) \in \mathcal{E}_{a, b}$ to $W_{c, d}=(0,0) \in \mathcal{E}_{c, d}$. Let

$$
\tilde{y}^{2}=\tilde{x}\left(\tilde{x}^{2}+\left(c^{2}+2 d\right) \tilde{x}+d^{2}\right)
$$

be the equation of $\mathcal{E}_{c, d}$ in coordinates $\tilde{x}, \tilde{y}$. According to 10, Sect. 1, p. 301, formula (1.13)], there exist a nonzero $u \in K$ and $\alpha, \beta, \gamma \in K$ such that the following conditions hold:

$$
\tilde{x} \circ \phi=u^{2} x+\alpha, \tilde{y} \circ \phi=u^{3} y+\beta u^{2} x+\gamma .
$$

Since $\phi$ sends $(0,0) \in \mathcal{E}_{a, b}(K)$ to $(0,0) \in \mathcal{E}_{c, d}(K)$, we have $\alpha=0=\gamma$. Since $\phi$ commutes with the multiplication by -1 , we conclude that $\beta=0$, i.e., $\phi$ is defined by the formulas

$$
\tilde{x} \circ \phi=u^{2} x, \tilde{y} \circ \phi=u^{3} y .
$$

Now [10, Sect. 1, p. 301, formula (1.14)] implies that

$$
u^{2}\left(a^{2}+2 b\right)=\left(c^{2}+2 d\right), u^{4} b^{2}=d^{2} .
$$

This proves our assertion in one direction. In order to prove it in the opposite direction, let us assume that (26) holds. Then the formulas (25) define an isomorphism $\phi: \mathcal{E}_{a, b} \cong \mathcal{E}_{c, d}$.

Remark 5.4. Let us look more closely at equalities (26). They may be rewritten as follows:

$$
u^{2}\left(a^{2}+2 b\right)=\left(c^{2}+2 d\right), d= \pm u^{2} b .
$$

It follows that $u^{2}\left(a^{2}+2 b\right)=\left(c^{2} \pm 2 u^{2} b\right)$, i.e., either

$$
d=u^{2} b, u^{2}\left(a^{2}+2 b\right)=\left(c^{2}+2 u^{2} b\right),
$$

and so

$$
u^{2} a^{2}=c^{2}, c= \pm u a,
$$

or

$$
d=-u^{2} b, u^{2}\left(a^{2}+2 b\right)=\left(c^{2}-2 u^{2} b\right),
$$


and therefore

$$
u^{2}\left(a^{2}+4 b\right)=c^{2}
$$

in the latter case $a^{2}+4 b$ is a square in $K$, which is not the case. So, we have

$$
d=u^{2} b, d c= \pm u a,
$$

and therefore $u= \pm c / a$, which implies that

We have

$$
b=\frac{d a^{2}}{c^{2}} .
$$

$$
\left(c^{2}+2 d\right)=\left( \pm \frac{c}{a}\right)^{2}\left(a^{2}+2 \cdot \frac{d a^{2}}{c^{2}}\right) u^{2}=u^{2}\left(a^{2}+2 b\right) .
$$

It follows that if $a$ is a fixed nonzero element of $K$ and $E$ is an elliptic curve over $K$ such that $E(K)$ contains a point of order 4 and exactly one point of order 2, then there exists exactly one (nonzero) $b \in K$ such that $E$ is $K$-isomorphic to $\mathcal{E}_{a, b}$. In particular, if we take $a=1 / 2$, then we get that there is exactly one $t \in K$ such that $E$ is $K$-isomorphic to $\mathcal{E}_{4, t}$.

\section{ElLiptic CURVES With POINTS OF ORDER 8}

Let $E$ be an elliptic curve over a field $K$ with $\operatorname{char}(K) \neq 2$. Suppose that $E(K)$ contains exactly one point of order 2 and two points of order 4 . Then there exist nonzero $a, b \in K$ such that $a^{2}+4 b$ is not a square in $K$ and $E$ is $K$-isomorphic to

$$
\mathcal{E}_{a, b}^{(4)}: y^{2}=x\left(x^{2}+\left(a^{2}+2 b\right) x+b^{2}\right) .
$$

6.1. Recall that $\mathcal{E}_{a, b}^{(4)}(K)$ contains exactly two points of order 4 , namely, $Q_{+}=$ $(-b, a b)$ and $Q_{-}=(-b,-a b)=-Q_{+}$. It follows that the existence of a point of order 8 in $\mathcal{E}_{a, b}^{(4)}(K)$ is equivalent to the divisibility by 2 of $Q_{+}$in $\mathcal{E}_{a, b}^{(4)}(K)$. By Theorem 4.4 and Remark 4.5. $Q_{+}$is divisible by 2 if and only if $(-b)-0$ is a square in $K$ and there exists $r \in K$ such that $r^{2}=x_{0}-\alpha$ and nonzero $\left(2 x_{0}+p\right)\left(x_{0}-\alpha\right)-2 y_{0} r$ is a square in $K$, where

$$
\alpha=0, p=a^{2}+2 b ; x_{0}=-b, y_{0}=a b .
$$

This means that $r^{2}=-b \neq 0$ and

$$
\left(-2 b+a^{2}+2 b\right)(-b)-2 a b r=a^{2} r^{2}+2 a r^{2} r=r^{2}\left(a^{2}+2 a r\right)=r^{2}\left((a+r)^{2}-r^{2}\right)
$$

is a nonzero square in $K$. It follows that $Q_{+}$is divisible by 2 in $\mathcal{E}_{a, b}^{(4)}(K)$ if and only if there exist nonzero $r, T \in K$ such that

$$
b=-r^{2}, T^{2}=(a+r)^{2}-r^{2} .
$$

If this is the case, then Theorem 4.4 gives us two distinct halves of $Q_{+}$in $\mathcal{E}_{a, b}^{(4)}(K)$, namely,

$$
\begin{gathered}
\mathcal{Q}_{r, T}=\left(x_{1}, y_{1}\right):=\left(-b+r T-\frac{a b}{r},-a b-(r+T)\left(r T-\frac{a b}{r}\right)\right) \\
\mathcal{Q}_{r,-T}=\left(x_{-1}, y_{-1}\right):=\left(-b-r T-\frac{a b}{r},-a b-(r-T)\left(-r T-\frac{a b}{r}\right)\right) ; \\
2 \mathcal{Q}_{r, T}=2 \mathcal{Q}_{r,-T}=Q_{+}, \mathcal{Q}_{r,-T}=\mathcal{Q}_{r, T}+W_{3} .
\end{gathered}
$$

This implies that both $\mathcal{Q}_{r, T}$ and $\mathcal{Q}_{r,-T}$ have order 8. 
Proposition 6.2. $\mathcal{E}_{a, b}^{(4)}(K)$ contains a point of order 8 if and only if there exist nonzero $r, t \in K$ such that $t \neq 0, \pm 1$ and

$$
b=-r^{2}, a=\frac{2 t^{2} r}{1-t^{2}} .
$$

Proof. Dividing the second equation in (27) by $r^{2}$, we get

$$
\left(\frac{T}{r}\right)^{2}=\left(\frac{a}{r}+1\right)^{2}-1
$$

Using the well-known rational parametrization of the hyperbola, we obtain that this equality is equivalent to the existence of a $t \in K, t \neq \pm 1$, such that

$$
\frac{a}{r}+1=\frac{1+t^{2}}{1-t^{2}}, \frac{T}{r}=\frac{2 t}{1-t^{2}} .
$$

This means that

$$
a=\frac{2 t^{2} r}{1-t^{2}}, T=\frac{2 t r}{1-t^{2}}
$$

for $t \in K, t \neq \pm 1$. Since $T \neq 0$, we have $t \neq 0$. We still need to find what does it mean in terms of $r, t$ that $a^{2}+4 b$ is not a square in $K$. We have

$$
a^{2}+4 b=\left(\frac{2 t^{2} r}{1-t^{2}}\right)^{2}+4\left(-r^{2}\right)=4 r^{2} \frac{2 t^{2}-1}{\left(1-t^{2}\right)^{2}} .
$$

This implies that $a^{2}+4 b$ is not a square in $K$ if and only if $t \neq \pm 1$ and $2 t^{2}-1$ is not a square.

Theorem 6.3. Let $E$ be an elliptic curve over a field $K$ with $\operatorname{char}(K) \neq 2$. Suppose that $E(K)$ contains exactly one point of order 2 . Then the following conditions are equivalent.

(i) $E(K)$ contains a cyclic subgroup of order 8.

(ii) There exists $t \in K$ such that

(1) $t \neq 0, \pm 1$,

(2) $2 t^{2}-1$ is not a square in $K$,

(3) $E$ is $K$-isomorphic to the elliptic curve

$$
\mathcal{E}_{t}^{(8)}: y^{2}=x\left(x^{2}+2 \frac{t^{4}+2 t^{2}-1}{\left(t^{2}-1\right)^{2}} x+1\right) .
$$

Proof. Combining Theorem 5.1 with Proposition 6.2. we conclude that the condition (i) is equivalent to the existence of nonzero $r, t \in K, t \neq \pm 1$, such that

$$
a=\frac{2 t^{2} r}{1-t^{2}}, b=-r^{2}
$$

enjoy the following properties.

(a) $a \neq 0$.

(b) $a^{2}+4 b$ is not a square in $K$.

(c) $E$ is isomorphic over $K$ to the elliptic curve

$$
\mathcal{E}_{a, b}^{(4)}: y^{2}=x\left(x^{2}+\left(a^{2}+2 b\right) x+b^{2}\right) .
$$


We notice that $a=0$ if and only if $t=0$; moreover,

$$
\begin{gathered}
b^{2}=\left(-r^{2}\right)^{2}=r^{4}, \\
a^{2}+2 b=2 r^{2} \frac{\left(t^{4}+2 t^{2}-1\right)}{\left(t^{2}-1\right)^{2}} .
\end{gathered}
$$

Consequently, the curve $E$ is $K$-isomorphic to the curve

$$
\mathcal{E}_{r, t}^{(8)}: y^{2}=x\left(x^{2}+2 r^{2} \frac{t^{4}+2 t^{2}-1}{\left(t^{2}-1\right)^{2}} x+r^{4}\right) .
$$

Dividing both sides by $r^{6}$ and using the change of variables $\tilde{x}=x / r^{2}, \tilde{y}=y / r^{3}$, we get the elliptic curve

$$
\mathcal{E}_{t}^{(8)}: \tilde{y}^{2}=\tilde{x}\left(\tilde{x}^{2}+2 \frac{t^{4}+2 t^{2}-1}{\left(t^{2}-1\right)^{2}} \tilde{x}+1\right)
$$

(where $t \neq 0, \pm 1$ ) that is $K$-isomorphic to $E$. It remains to notice that $a^{2}+4 b$ is not a square in $K$ if and only if $2 t^{2}-1$ is not a square.

Example 6.4. Let us describe explicitly points of order 8 in $\mathcal{E}_{t}^{(8)}(K)$. Notice that $\mathcal{E}_{t}^{(8)}=\mathcal{E}_{a, b}^{(4)}$ with

$$
a=\frac{2 t^{2}}{1-t^{2}}, \quad b=-1, r=1, T=\frac{2 t}{1-t^{2}} .
$$

In particular, $\mathcal{E}_{t}^{(8)}(K)$ contains a point $Q_{+}=(-b, a b)=\left(1,-\frac{2 t^{2}}{1-t^{2}}\right)$ of order 4 . Clearly, the conditions (27) hold. Then (28) give us two distinct order 8 points in $\mathcal{E}_{t}^{(8)}(K)$, namely,

$$
\begin{gathered}
\mathcal{Q}_{r, T}=\left(1+\frac{2 t}{1-t^{2}}+\frac{2 t^{2}}{1-t^{2}}, \frac{2 t^{2}}{1-t^{2}}-\left(1+\frac{2 t}{1-t^{2}}\right)\left(\frac{2 t}{1-t^{2}}+\frac{2 t^{2}}{1-t^{2}}\right)\right) \\
=\left(\frac{t+1}{1-t}, \frac{-2 t}{(1-t)^{2}}\right), \\
\mathcal{Q}_{r,-T}=\left(1-\frac{2 t}{1-t^{2}}+\frac{2 t^{2}}{1-t^{2}}, \frac{2 t^{2}}{1-t^{2}}-\left(1-\frac{2 t}{1-t^{2}}\right)\left(-\frac{2 t}{1-t^{2}}+\frac{2 t^{2}}{1-t^{2}}\right)\right) \\
=\left(\frac{1-t}{1+t}, \frac{2 t}{(1+t)^{2}}\right) ; \\
2 \mathcal{Q}_{r, T}=2 \mathcal{Q}_{r,-T}=Q_{+}, \mathcal{Q}_{r,-T}=\mathcal{Q}_{r, T}+W_{3} .
\end{gathered}
$$

So, $\mathcal{E}_{t}^{(8)}(K)$ contains exactly four points of order 8 , namely,

$$
\left(\frac{1+t}{1-t}, \frac{-2 t}{(1-t)^{2}}\right),\left(\frac{1-t}{1+t}, \frac{2 t}{(1+t)^{2}}\right)
$$

and their negatives

$$
\left(\frac{1+t}{1-t}, \frac{2 t}{(1-t)^{2}}\right),\left(\frac{1-t}{1+t}, \frac{-2 t}{(1+t)^{2}}\right) .
$$


Remark 6.5. D. Kubert [5] described another family of elliptic curves

$$
\mathcal{E}_{8, d}: y^{2}+(1-c) x y-b y=x^{3}-b x^{2}
$$

with point $Q=(0,0)$ of order 8 , where

$$
c=\frac{(2 d-1)(d-1)}{d}, \quad b=(2 d-1)(d-1) .
$$

Completing the square on the left-hand side of the equation, we get

$$
\left(y+\frac{(1-c) x-b}{2}\right)^{2}=x^{3}-b x^{2}+\left(\frac{(1-c) x-b}{2}\right)^{2} .
$$

The curve given by the latter equation is isomorphic to

$$
y^{2}=x^{3}+\left(\left(\frac{1-c}{2}\right)^{2}-b\right) x^{2}-\frac{(1-c) b}{2} x+\frac{b^{2}}{4} .
$$

Since

we get

$$
\frac{1-c}{2}=\frac{-2 d^{2}+4 d-1}{2 d}
$$

$$
\begin{array}{r}
y^{2}=x^{3}+\left(\frac{-4 d^{4}-4 d^{3}+16 d^{2}-8 d+1}{4 d^{2}}\right) x^{2} \\
-\frac{\left(-2 d^{2}+4 d-1\right)(2 d-1)(d-1)}{2 d} x+\frac{(2 d-1)^{2}(d-1)^{2}}{4} .
\end{array}
$$

Multiplying both sides of the above equation by $d^{6}$ and replacing $y d^{3}$ by $y$ and $x d^{2}$ by $x$, we get the equation with polynomial coefficients

$$
\begin{array}{r}
y^{2}=x^{3}+\left(\frac{-4 d^{4}-4 d^{3}+16 d^{2}-8 d+1}{4}\right) x^{2} \\
-\frac{\left(-2 d^{2}+4 d-1\right)(2 d-1)(d-1) d^{3}}{2} x+\frac{d^{6}(2 d-1)^{2}(d-1)^{2}}{4} .
\end{array}
$$

The polynomial on the right can be factored as follows:

$$
\left(x-\left(d^{4}-d^{3}\right)\right)\left(x^{2}+\frac{1-8 d(d-1)^{2}}{4} x-\frac{\left.d^{3}(d-1)(2 d-1)^{2}\right)}{4}\right) .
$$

Now using the change of variables $x-\left(d^{4}-d^{3}\right) \rightarrow x$, we obtain that

$$
y^{2}=x\left(x^{2}+\frac{8 d^{4}-16 d^{3}+16 d^{2}-8 d+1}{4} x+d^{4}(d-1)^{4}\right) .
$$

Dividing both sides by $d^{6}(d-1)^{6}$ and using the change of variables $\tilde{x}=x / d^{2}(d-1)^{2}$, $\tilde{y}=y / d^{3}(d-1)^{3}$, we get the elliptic curve

$$
\tilde{y}^{2}=\tilde{x}\left(\tilde{x}^{2}+\frac{8 d^{4}-16 d^{3}+16 d^{2}-8 d+1}{4 d^{2}(d-1)^{2}} \tilde{x}+1\right) .
$$

It is easy to verify that

$$
\frac{8 d^{4}-16 d^{3}+16 d^{2}-8 d+1}{4 d^{2}(d-1)^{2}}=2 \frac{t^{4}+2 t^{2}-1}{\left(t^{2}-1\right)^{2}},
$$

where $t=2 d-1$. This implies that $\mathcal{E}_{8, d}$ is isomorphic over $K$ to $\mathcal{E}_{t}^{(8)}$ with $t=2 d-1$. 
Theorem 6.6. Let $K$ be a field with $\operatorname{char}(K) \neq 2$. Let $s, t \in K$ be nonzero elements of $K$ such that $s, t \neq \pm 1$ and $2 t^{2}-1,2 s^{2}-1$ are not squares in $K$. If $K$ does not contain a primitive 4 th root of 1 , then the elliptic curves $\mathcal{E}_{s}^{(8)}$ and $\mathcal{E}_{t}^{(8)}$ are $K$ isomorphic if and only if $s= \pm t$ or $s^{2}+t^{2}=2 s^{2} t^{2}$. If $K$ contains a primitive 4 th root of 1 , then $\mathcal{E}_{s}^{(8)}$ and $\mathcal{E}_{t}^{(8)}$ are $K$-isomorphic if and only if $s= \pm t, s^{2}+t^{2}=2 s^{2} t^{2}$, or $s^{4} t^{4}+2 s^{2}+2 t^{2}=4 s^{2} t^{2}+1$.

Proof. It follows from Theorem 5.3 that $\mathcal{E}_{s}^{(8)}$ and $\mathcal{E}_{t}^{(8)}$ are $K$-isomorphic if and only if there exists $u \in K$ such that $u^{4}=1$ and

$$
\frac{s^{4}+2 s^{2}-1}{\left(s^{2}-1\right)^{2}}=u^{2} \frac{t^{4}+2 t^{2}-1}{\left(t^{2}-1\right)^{2}} .
$$

If $K$ does not contain a primitive 4 th root of 1 , then the latter equality is possible only if

$$
\frac{s^{4}+2 s^{2}-1}{\left(s^{2}-1\right)^{2}}=\frac{t^{4}+2 t^{2}-1}{\left(t^{2}-1\right)^{2}},
$$

which takes place if and only if one of the equalities $s= \pm t$ or $s^{2}+t^{2}=2 s^{2} t^{2}$ is valid. If $K$ contains a primitive 4 th root of 1 , then we have

$$
\frac{s^{4}+2 s^{2}-1}{\left(s^{2}-1\right)^{2}}= \pm \frac{t^{4}+2 t^{2}-1}{\left(t^{2}-1\right)^{2}}
$$

which holds if and only if $s= \pm t, s^{2}+t^{2}=2 s^{2} t^{2}$, or $s^{4} t^{4}+2 s^{2}+2 t^{2}=4 s^{2} t^{2}+1$.

\section{ElLiptic CURVES With POINT OF ORDER 6}

Let $K$ be a field with $\operatorname{char} K \neq 0$. Let $E$ be an elliptic curve over $K$ defined by the equation

$$
y^{2}=f(x)
$$

where $f(x) \in K[x]$ is a monic cubic polynomial without repeated roots.

Example 7.1. Let $t \in K \backslash 0,-4,1 / 2$. Let us consider the elliptic curve

$$
\mathcal{E}_{t}^{(6)}: y^{2}=\left(x^{2}+\left(t^{2}+2 t\right) x+t^{2}\right)(x+1)
$$

over $K$. (We assume that $t \notin\{0,-4,1 / 2\}$ to exclude the case when the cubic polynomial has a repeated root.) The group $E(K)$ contains the point $W_{3}=(-1,0)$ of order 2 and the point $P=(0, t)$. Let us put

$$
y_{0}=t, r=1, T=t .
$$

Then the curve $\mathcal{E}_{t}^{(6)}$ coincides with the elliptic curve (23) from Theorem 4.6 According to this theorem,

$$
\mathcal{Q}_{r, T}=\left(r T-\frac{y_{0}}{r},-y_{0}-(r+T)\left(r T-\frac{y_{0}}{r}\right)\right)
$$

and

$$
\mathcal{Q}_{r,-T}=\left(-r T-\frac{y_{0}}{r},-y_{0}-(r-T)\left(-r T-\frac{y_{0}}{r}\right)\right)
$$

are distinct points of $E(K)$ that satisfy

$$
2 \mathcal{Q}_{r, T}=2 \mathcal{Q}_{r,-T}=P, \mathcal{Q}_{r,-T}=\mathcal{Q}_{r, T}+W_{3}
$$

In our case

$$
r T=t, \frac{y_{0}}{r}=t, r T-\frac{y_{0}}{r}=0,-r T-\frac{y_{0}}{r}=-2 t .
$$


FAMILIES OF ELLIPTIC CURVES WITH RATIONAL TORSION POINTS OF EVEN ORDER1

This implies that

$$
\begin{array}{r}
\mathcal{Q}_{r, T}=\left(0,-y_{0}\right)=(0,-t)=-P, \mathcal{Q}_{r, T}+W_{3}=\mathcal{Q}_{r,-T} \\
=(-2 t,-t-(1-t)(-2 t))=\left(-2 t, t-2 t^{2}\right) .
\end{array}
$$

Since

$$
\mathcal{Q}_{r, T}=-P, 2 \mathcal{Q}_{r, T}=-P
$$

$P$ and $\mathcal{Q}_{r, T}$ have order 3 while $\mathcal{Q}_{r,-T}=\mathcal{Q}_{r, T}+W_{3}$ has order 6 . So, the point $(0, t) \in \mathcal{E}_{t}^{(6)}(K)$ has order 3 and the point $\left(-2 t, t-2 t^{2}\right) \in \mathcal{E}_{t}^{(6)}(K)$ has order 6.

Notice that $\mathcal{E}_{t}^{(6)}(K)$ contains exactly one point of order 2 if and only if the discriminant $\left(t^{2}+2 t\right)^{2}-4 t^{2}=t^{2}\left(t^{2}+4 t\right)$ of $x^{2}+\left(t^{2}+2 t\right) x+t^{2}$ is not a square, i.e., if and only if $t^{2}+4 t$ is not a square.

Theorem 7.2. The following conditions on $E$ are equivalent.

(i) $E(K)$ contains a point of order 6 .

(ii) There exists $t \in K \backslash\{0,-4,1 / 2\}$ such that $E$ is isomorphic over $K$ to the elliptic curve

$$
\mathcal{E}_{t}^{(6)}: y^{2}=(x+1)\left(x^{2}+\left(t^{2}+2 t\right) x+t^{2}\right) .
$$

Proof. Suppose $E(K)$ contains a point of order 6 . This means that $E(K)$ contains a point of order 2 and a point of order 3 . The existence of a point of order 2 in $E(K)$ means that $f(x)$ has a root in $K$ say, $\alpha$ and one may represent $f(x)$ as a product

$$
f(x)=g(x)(x-\alpha) \in K[x]
$$

where

$$
g(x)=x^{2}+p x+q \in K[x]
$$

is a monic quadratic polynomial without repeated roots such that our $\alpha \in K$ is not a root of $g(x)$. Then $W_{3}=(\alpha, 0) \in E(K)$ is a point of order 2 .

Let $P=\left(x_{0}, y_{0}\right) \in E(K)$ be a point of order 3 . Since $3 \neq 2$, we have $y_{0} \neq 0$. Using the change of variables $x \rightarrow x-x_{0}$ (and replacing $\alpha$ by $\alpha-x_{0}$ and $g(x)$ by $\left.g\left(x-x_{0}\right)\right)$, we may and will assume that $x_{0}=0$, i.e., $P=\left(0, y_{0}\right)$. Since $P$ has order 3 , it lies in $2 E(K)$, because $2(-P)=P$. Let us apply the last assertion of Theorem 4.6 to $P=\left(0, y_{0}\right)$ and $Q=-P=\left(0,-y_{0}\right)$. We obtain that there exist $r \in K$ and a nonzero $T \in K$ such that

$$
\begin{aligned}
& r^{2}=-\alpha, g(x)=x^{2}+p x+q=x^{2}+\left(T^{2}+2 \frac{y_{0}}{r}\right) x+\left(\frac{y_{0}}{r}\right)^{2}, \\
& \left(0,-y_{0}\right)=Q=\mathcal{Q}_{r, T}=\left(r T-\frac{y_{0}}{r},-y_{0}-(r+T)\left(r T-\frac{y_{0}}{r}\right)\right) .
\end{aligned}
$$

Looking at the $x$-coordinates, we see that

$$
r T-\frac{y_{0}}{r}=0, r T=\frac{y_{0}}{r}, y_{0}=r^{2} T,
$$

and therefore

$$
g(x)=x^{2}+\left(T^{2}+2 r T\right) x+(r T)^{2}, f(x)=\left(x^{2}+\left(T^{2}+2 r T\right) x+(r T)^{2}\right)\left(x+r^{2}\right)
$$

and the equation for $E$ is

$$
E: y^{2}=f(x)=\left(x^{2}+\left(T^{2}+2 r T\right) x+(r T)^{2}\right)\left(x+r^{2}\right) .
$$


Dividing both sides of this equation by $r^{6}$, and making a change of variables $\tilde{x}=$ $x / r^{2}, \tilde{y}=y / r^{3}$, we obtain that $E$ is isomorphic to the elliptic curve

$$
\mathcal{E}_{t}^{(6)}: \tilde{y}^{2}=(\tilde{x}+1)\left(\tilde{x}^{2}+\left(t^{2}+2 t\right) \tilde{x}+t^{2}\right)
$$

with $t=T / r \neq 0$. Since the polynomial $(\tilde{x}+1)\left(\tilde{x}^{2}+\left(t^{2}+2 t\right) \tilde{x}+t^{2}\right)$ has no multiple roots, we conclude that $t \notin\{-4,0,1 / 2\}$.

The converse assertion follows from Example 7.1 .

Remark 7.3. In Theorem 7.2 we do not assume that $\operatorname{char}(K) \neq 3$ !

Example 7.4. Let $K=\mathbb{F}_{3}$ be the 3 -element field. Then there is exactly one element $t$ in $\mathbb{F}_{3} \backslash\{0,-4\}$, namely $t=1$. It follows from Theorem 7.2 that $E$ is an elliptic curve over $K$ such that $E\left(\mathbb{F}_{3}\right)$ contains a point of order 6 if and only if $E$ is $\mathbb{F}_{3}$-isomorphic to

$$
\mathcal{E}_{1}^{(6)}: y^{2}=(x+1)\left(x^{2}+1\right) .
$$

Let us consider the curve $\mathcal{E}_{1}^{(6)}$. It contains an $\mathbb{F}_{3}$-point of order 6 , namely, $Q=$ $\left(-2 t, t-2 t^{2}\right)=(-2,-1)$. Hence the whole group $\mathcal{E}_{1}^{(6)}\left(\mathbb{F}_{3}\right)$ has order divisible by 6. On the other hand, by Hasse's bound, the order of $\mathcal{E}_{1}^{(6)}\left(\mathbb{F}_{3}\right)$ does not exceed $3+2 \sqrt{3}+1<6 \cdot 2$. This implies that $\mathcal{E}_{1}^{(6)}\left(\mathbb{F}_{3}\right)$ has order 6 and therefore coincides with the cyclic group of order 6 generated by $Q$.

Remark 7.5. D. Kubert described another family of elliptic curves

$$
\mathcal{E}_{6, t}: y^{2}+(1-c) x y-\left(c+c^{2}\right) y=x^{3}-\left(c+c^{2}\right) x^{2}
$$

with point $Q=(0,0)$ of order 6 . The equation for $\mathcal{E}_{6, c}$ is equivalent to

$$
\begin{array}{r}
y^{2}+2 y \frac{(1-c) x-\left(c+c^{2}\right)}{2}+\frac{\left((1-c) x-\left(c+c^{2}\right)\right)^{2}}{4} \\
=x^{3}-\left(c+c^{2}\right) x^{2}+\frac{\left((1-c) x-\left(c+c^{2}\right)\right)^{2}}{4} .
\end{array}
$$

The left-hand side is equal to $\left(y+\left((1-c) x-\left(c+c^{2}\right)\right) / 2\right)^{2}$ while the right-hand side splits into the product

$$
(x-c)\left(x^{2}+\frac{-3 c^{2}-2 c+1}{4} x-\frac{c^{3}+2 c^{2}+c}{4}\right) .
$$

After the change of variables $\tilde{x}=x-\left(c^{2}+c\right), \tilde{y}=y+\left((1-c) x-\left(c+c^{2}\right)\right) / 2$, we get the equation

$$
\tilde{y}^{2}=\left(\tilde{x}+c^{2}\right)\left(\tilde{x}^{2}+\frac{5 c^{2}+6 c+1}{4} \tilde{x}+\frac{c^{2}(c+1)^{2}}{4}\right) .
$$

Dividing both sides by $c^{6}$, we get the equation

$$
\left(\frac{\tilde{y}}{c^{3}}\right)^{2}=\left(\frac{\tilde{x}}{c^{2}}+1\right)\left(\left(\frac{\tilde{x}}{c^{2}}\right)^{2}+\frac{5 c^{2}+6 c+1}{4 c^{2}}\left(\frac{\tilde{x}}{c^{2}}\right)+\frac{(c+1)^{2}}{4 c^{2}}\right) .
$$

Now the change of variables $\bar{x}=\tilde{x} / c^{2}, \bar{y}=\tilde{y} / c^{3}$ gives us the equation

$$
\bar{y}^{2}=(\bar{x}+1)\left(\bar{x}^{2}+\frac{5 c^{2}+6 c+1}{4 c^{2}} \bar{x}+\left(\frac{c+1}{2 c}\right)^{2}\right),
$$

which is nothing else but the equation of $\mathcal{E}_{t}^{(6)}$ with $t=(c+1) / 2 c$. 


\section{Elliptic CURVES With POINT OF ORDER 12}

Let $K$ be a field with $\operatorname{char}(K) \neq 2$, and let $t \in K \backslash\{0,-4,1 / 2\}$ be such that $t^{2}+4 t$ is not a square in $K$. Let us consider the elliptic curve

$$
E:=\mathcal{E}_{t}^{(6)}: y^{2}=g(x)(x+1)
$$

where

$$
g(x)=x^{2}+\left(t^{2}+2 t\right) x+t^{2}
$$

is a quadratic irreducible polynomial over $K$. Then $W=W_{3}=(-1,0)$ is the only point of order 2 in $E(K)$. We know that $E(K)$ contains a point of order 3 . Hence $E(K)$ contains a point of order 12 if and only if $W$ is divisible by 2 in $E(K)$. This is equivalent to the condition that $-1-\mathbf{x}$ is a square in the quadratic extension

$$
K_{g}=K[x] / g(x) K[x]
$$

of $K$, i.e., there exist $u, v \in K$ such that

$$
-1-\mathbf{x}=(u \mathbf{x}+v)^{2} \in K_{g} .
$$

Clearly, such $u \neq 0$. (Here $\mathbf{x}$ is the image of $x$ in $K_{g}$.) In other words, $W$ is divisible by 2 in $E(K)$ if and only if there exist $u, v \in K$ such that $(u x+v)^{2}-(-1-x)$ is divisible by $g(x)$. The latter condition means that there exist $u, v \in K$ such that

$$
u^{2} x^{2}+(2 u v+1) x+\left(v^{2}+1\right)=(u x+v)^{2}+x+1
$$

coincides with

$$
u^{2} g(x)=u^{2} x^{2}+u^{2}\left(t^{2}+2 t\right) x+u^{2} t^{2},
$$

i.e.,

$$
2 u v+1=u^{2}\left(t^{2}+2 t\right), v^{2}+1=u^{2} t^{2} .
$$

Subtracting one equation from the other, we obtain that

$$
v^{2}-2 u v=-2 t u^{2} .
$$

Dividing both sides by $u^{2}$ and putting $\lambda=v / u$, we get

$$
\lambda^{2}-2 \lambda=-2 t,
$$

which gives us

$$
t=\frac{2 \lambda-\lambda^{2}}{2}, v=\lambda u
$$

This implies that $\lambda \neq 0$, since $t \neq 0$ and therefore $v \neq 0$. We have

$$
(\lambda u)^{2}+1=u^{2}\left(\frac{2 \lambda-\lambda^{2}}{2}\right)^{2},
$$

which means that

$$
1=u^{2}\left(\left(\frac{2 \lambda-\lambda^{2}}{2}\right)^{2}-\lambda^{2}\right)=u^{2} \lambda^{2}\left(\left(\frac{2-\lambda}{2}\right)^{2}-1\right) .
$$

Consequently,

$$
1=v^{2}\left(\left(1-\frac{\lambda}{2}\right)^{2}-1\right) .
$$

Putting $s=1-\lambda / 2$ and $\mu=1 / v$, we get the equation for a hyperbola in $(s, \mu)$ coordinates.

$$
\mu^{2}=s^{2}-1
$$


Using the standard parametrization

$$
s=\frac{1+T^{2}}{1-T^{2}}, \quad \mu=\frac{2 T}{1-T^{2}}
$$

of this hyperbola, we obtain

$$
\begin{array}{r}
\lambda=\frac{4 T^{2}}{T^{2}-1}, \quad t=\frac{2 \lambda-\lambda^{2}}{2}=-\frac{4 T^{2}\left(T^{2}+1\right)}{\left(T^{2}-1\right)^{2}}, \\
t^{2}+2 t=\frac{8 T^{2}\left(T^{2}+1\right)\left(T^{4}+4 T^{2}-1\right)}{\left(T^{2}-1\right)^{4}},
\end{array}
$$

and the equation of $E$ takes the form

$$
y^{2}=(x+1)\left(x^{2}+\frac{8 T^{2}\left(T^{2}+1\right)\left(T^{4}+4 T^{2}-1\right)}{\left(T^{2}-1\right)^{4}} x+\frac{16 T^{4}\left(T^{2}+1\right)^{2}}{\left(T^{2}-1\right)^{4}}\right) .
$$

The condition $t \in K \backslash\{0,-4,1 / 2\}$ is equivalent to $T \notin\{0, \pm 1, \pm \sqrt{-1}\}, 3 T^{2}-1 \neq 0$, and $3 T^{2}+1 \neq 0$. Since

$$
t^{2}+4 t=\frac{16 T^{2}\left(T^{2}+1\right)\left(3 T^{2}-1\right)}{\left(T^{2}-1\right)^{4}}
$$

$t^{2}+4 t$ is not a square in $K$ if and only if $\left(T^{2}+1\right)\left(3 T^{2}-1\right)$ is not a square in $K$.

We have proved the following statement.

Theorem 8.1. The following conditions on $E$ are equivalent.

(i) $E(K)$ contains exactly one point of order 2 and a point of order 12 .

(ii) There exists $T \in K \backslash\{0, \pm 1, \pm \sqrt{-1}\}$ such that $3 T^{2}+1 \neq 0,\left(T^{2}+1\right)\left(3 T^{2}-1\right)$ is not a square, and $E$ is isomorphic over $K$ to the elliptic curve

$$
\mathcal{E}_{T}^{(12)}: y^{2}=(x+1)\left(x^{2}+\frac{8 T^{2}\left(T^{2}+1\right)\left(T^{4}+4 T^{2}-1\right)}{\left(T^{2}-1\right)^{4}} x+\frac{16 T^{4}\left(T^{2}+1\right)^{2}}{\left(T^{2}-1\right)^{4}}\right) .
$$

Remark 8.2. $\mathcal{E}_{T}^{(12)}(K)$ contains points $\left(0, \pm 4 T^{2}\left(T^{2}+1\right) /\left(T^{2}-1\right)^{2}\right)$ of order 3 and exactly two points of order 4 . Applying the explicit formulas from Theorem 3.3 to $\left(x_{0}, y_{0}\right)=(-1,0)$ and taking into account that $r=x_{0}-\alpha=0, \mathbf{r}=u \mathbf{x}+v$,

$$
\begin{aligned}
\operatorname{Norm}(\mathbf{r}) & =u^{2} \operatorname{Norm}(\mathbf{x})+u v \operatorname{Tr}(\mathbf{x})+v^{2}=t^{2} u^{2}-\left(t^{2}+2 t\right) u v+v^{2}, \\
\operatorname{Tr}(\mathbf{r}) & =u \operatorname{Tr}(\mathbf{x})+2 v=-\left(t^{2}+2 t\right) u+2 v,
\end{aligned}
$$

we get the following two points of order 4 on $\mathcal{E}_{T}^{(12)}$ :

$$
\begin{gathered}
\left(t^{2} u^{2}-\left(t^{2}+2 t\right) u v+v^{2},\left(t^{2} u^{2}-\left(t^{2}+2 t\right) u v+v^{2}\right)\left(-\left(t^{2}+2 t\right) u+2 v\right)\right), \\
\left(t^{2} u^{2}-\left(t^{2}+2 t\right) u v+v^{2},-\left(t^{2} u^{2}-\left(t^{2}+2 t\right) u v+v^{2}\right)\left(-\left(t^{2}+2 t\right) u+2 v\right)\right),
\end{gathered}
$$

where

$$
\begin{array}{r}
t=-\frac{4 T^{2}\left(T^{2}+1\right)}{\left(T^{2}-1\right)^{2}}, t^{2}+2 t=\frac{8 T^{2}\left(T^{2}+1\right)\left(T^{4}+4 T^{2}-1\right)}{\left(T^{2}-1\right)^{4}}, \\
v=\frac{1}{\mu}=\frac{1-T^{2}}{2 T}, u=\frac{v}{\lambda}=-\frac{\left(T^{2}-1\right)^{2}}{8 T^{3}} .
\end{array}
$$

Substituting these expressions for $t, u$, and $v$ in the above formulas, we obtain the order 4 points

$$
\left(-\frac{3 T^{2}+1}{T^{2}-1},-\frac{8 T^{3}\left(3 T^{2}+1\right)}{\left(T^{2}-1\right)^{3}}\right),\left(-\frac{3 T^{2}+1}{T^{2}-1}, \frac{8 T^{3}\left(3 T^{2}+1\right)}{\left(T^{2}-1\right)^{3}}\right) .
$$

Remark 8.3. In Theorem 8.1 we do not assume that $\operatorname{char}(K) \neq 3$ ! 


\section{ElLiptic CURVES With RATIONAL POINTS OF ORDER 10}

Let $E$ be an elliptic curve over a field $K$ with $\operatorname{char}(K) \neq 2$. Suppose that $E(K)$ contains exactly one point of order 2 and a point $P$ of order 5 . We may assume that the first coordinate of $P$ is 0 . Since $P$ is divisible by 2 , Theorem 4.6 and Remark 4.7 tell us that $E$ is $K$-isomorphic to the elliptic curve

$$
E_{t, y_{0}}: y^{2}=(x+1)\left(x^{2}+\left(t^{2}+2 y_{0}\right) x+y_{0}^{2}\right),
$$

where $t^{2}+4 y_{0} \neq 0$ and $1-t^{2}-2 y_{0}+y_{0}^{2} \neq 0$. We may also assume that $P=\left(0, y_{0}\right)$. Clearly $P$ has order 5 if and only if there exists a $Q \in E(K)$ such that $2 P=-Q$ and $2 Q=P$. Using equation (20) with $p=t^{2}+2 y_{0}$ and $q=y_{0}^{2}$ and the equality $x(Q)=t-y_{0}$ obtained in Remark 4.7 we can write the equalities $2 P=-Q$ and $2 Q=P$ in the following equivalent form:

$$
\frac{\left(t^{2}+2 y_{0}-y_{0}^{2}\right)^{2}}{4 y_{0}^{2}}-1=t-y_{0} .
$$

Multiplying both sides by $y_{0}^{2}$ and removing parentheses, we get

$$
\begin{gathered}
t^{4}+y_{0}^{4}+4 t^{2} y_{0}-4 t y_{0}^{2}-2 t^{2} y_{0}^{2}=0, \\
\left(t^{2}-y_{0}^{2}\right)^{2}+4 t y_{0}\left(t-y_{0}\right)=0 .
\end{gathered}
$$

If $t=y_{0}$, then by Remark 4.7 we obtain $Q_{1, t}=\left(0,-y_{0}\right), P=-Q_{1, t}$, and so $3 P=0$, which is impossible. Dividing both sides of the above equation by $t-y_{0}$, we get

$$
\left(t-y_{0}\right)\left(t+y_{0}\right)^{2}+4 t y_{0}=0 .
$$

To obtain a rational parametrization of this equation, let us put $y_{0}=u t$ with $u \in K \backslash\{0,1\}$. Then

$$
t^{3}(1-u)(1+u)^{2}+4 t^{2} u=0
$$

hence

Since

$$
t=\frac{4 u}{(u-1)(u+1)^{2}}, y_{0}=\frac{4 u^{2}}{(u-1)(u+1)^{2}} .
$$

$$
t^{2}+2 y_{0}=\frac{16 u^{2}}{(u-1)^{2}(u+1)^{4}}+\frac{8 u^{2}}{(u-1)(u+1)^{2}}=\frac{8 u^{2}\left(u^{3}+u^{2}-u+1\right)}{(u-1)^{2}(u+1)^{4}},
$$

equation (31) transforms to

$$
y^{2}=(x+1)\left(x^{2}+\frac{8 u^{2}\left(u^{3}+u^{2}-u+1\right)}{(u-1)^{2}(u+1)^{4}} x+\frac{16 u^{4}}{(u-1)^{2}(u+1)^{4}}\right) .
$$

Since

$$
t^{2}+4 y_{0}=\frac{16 u^{3}\left(u^{2}+u-1\right)}{(u-1)^{2}(u+1)^{4}} \text { and } 1-t^{2}-2 y_{0}+y_{0}^{2}=\frac{\left(u^{2}-4 u-1\right)(u-1)}{(u+1)^{3}},
$$

the conditions $t^{2}+4 y_{0} \neq 0$ and $1-t^{2}-2 y_{0}+y_{0}^{2} \neq 0$ are equivalent to $u \neq 0, u \neq \pm 1$, $u^{2}+u \neq 1$, and $u^{2}-4 u-1 \neq 0$. Moreover, $t^{2}+4 y_{0}$ is not a square if and only if $u\left(u^{2}+u-1\right)$ is not a square.

We have proved the following statement.

Theorem 9.1. The following conditions on $E$ are equivalent.

(i) $E(K)$ contains exactly one point of order 2 and a point of order 10. 
(ii) There exists $u \in K \backslash\{0, \pm 1,(-1 \pm \sqrt{5}) / 2,2 \pm \sqrt{5}\}$ such that $u\left(u^{2}+u-1\right)$ is not a square and $E$ is isomorphic over $K$ to the elliptic curve

$$
\mathcal{E}_{u}^{(10)}: y^{2}=(x+1)\left(x^{2}+\frac{8 u^{2}\left(u^{3}+u^{2}-u+1\right)}{(u-1)^{2}(u+1)^{4}} x+\frac{16 u^{4}}{(u-1)^{2}(u+1)^{4}}\right) \text {. }
$$

Remark 9.2. $\mathcal{E}_{u}^{(10)}(K)$ contains a point $\left(0,4 u^{2} /(u-1)(u+1)^{2}\right)$ of order 5 and exactly one point $(-1,0)$ of order 2 .

Remark 9.3. In Theorem 9.1 we do not assume that $\operatorname{char}(K) \neq 5$ !

\section{Elliptid CURVES IN CHARACTERISTIC 2}

In this section we assume that $K$ is a field of characteristic 2 and $\bar{K}$ is its algebraic closure. It is known [9, Appendix A] that $E$ is ordinary (i.e., $E(\bar{K})$ contains a point of order 2) if and only if $j(E) \neq 0$. Let $E$ be an elliptic curve over $K$ defined by the equation

$$
y^{2}+x y=x^{3}+a_{2} x^{2}+a_{6},
$$

where

$$
a_{2}, a_{6} \in K ; a_{6} \neq 0, j(E)=\frac{1}{a_{6}} \neq 0
$$

As above, $E$ has the only one infinite point $\infty=(0: 1: 0)$, which is taken as the zero of the group law on $E$. It is known [9, Appendix A] that $E$ is ordinary. In addition, every ordinary elliptic curve $\tilde{E}$ over $K$ is isomorphic to $E$ for suitable $a_{2} \in K$ and $a_{6}=1 / j(\tilde{E})$ [9, Appendix A], [11, Sect. 2.8]. If $P=\left(x_{0}, y_{0}\right) \in E(\bar{K})$, then

$$
-P=\left(x_{0}, y_{0}+x_{0}\right) \in E(\bar{K}) .
$$

It follows that

$$
W_{3}=\left(0, \sqrt{a_{6}}\right)=\left(0, \frac{1}{\sqrt{j(E)}}\right) \in E(\bar{K})
$$

is the only point of order 2 in $E(\bar{K})$.

This implies the following assertion (that may be also extracted from [4]).

Proposition 10.1. An elliptic curve $E$ over a field $K$ of characteristic 2 has a $K$-rational point of order 2 if and only if $j(E)$ is a nonzero square in $K$.

Our first goal is to find explicitly both halves of $P$. So, let $Q=\left(x_{1}, y_{1}\right) \in E(\bar{K})$ with $2 Q=P$. Clearly, $Q \neq W$, i.e., $x_{1} \neq 0$, and therefore the tangent line $\mathcal{L}$ to $E$ at $Q$ is not vertical, i.e, may be written in the form

$$
y=l x+m ; l, m \in \bar{K} .
$$

(If $Q \in E(K)$, then both $l$ and $m$ lie in $K$.) Since $2 Q=P$, the line $\mathcal{L}$ contains $-P$. Restricting (as usual) the equation of $E$ to $\mathcal{L}$, we get the equation

$$
g(x)=x^{3}+a_{2} x+a_{6}+(l x+m)^{2}+x(l x+m)=0 .
$$

We know that $x=x_{1}$ is a multiple root of the monic cubic polynomial $g(x)$ and $x=x_{0}$ is a root of $g(x)$. This implies that

$$
g(x)=\left(x+x_{1}\right)^{2}\left(x+x_{0}\right) .
$$

It follows that

$$
x^{3}+\left(a_{2}+l^{2}+l\right) x^{2}+m x+\left(a_{6}+m^{2}\right)=x^{3}+x_{0} x^{2}+x_{1}^{2} x+x_{1}^{2} x_{0},
$$


FAMILIES OF ELLIPTIC CURVES WITH RATIONAL TORSION POINTS OF EVEN ORDER7

i.e,

$$
\left(a_{2}+l^{2}+l\right)=x_{0}, m=x_{1}^{2},\left(a_{6}+m^{2}\right)=x_{1}^{2} x_{0} .
$$

This implies that

$$
l^{2}+l=x_{0}+a_{2} .
$$

Since $-P=\left(x_{0}, y_{0}+x_{0}\right)$ lies on $L: y=l x+m$, we obtain

(33) $m=\left(y_{0}+x_{0}\right)-l x_{0}=y_{0}+(l+1) x_{0}, x_{1}=\sqrt{m}, y_{1}=l x_{1}+m=l \sqrt{m}+m$.

If we replace $l$ by $l+1$, then we should replace $m$ by $m+x_{0}$ and $x_{1}$ by $x_{1}+\sqrt{x_{0}}$. We also obtain the following formulas:

$$
\sqrt{a_{6}}+m=x_{1} \sqrt{x_{0}}, \sqrt{a_{6}}+y_{0}+(l+1) x_{0}=x_{1} \sqrt{x_{0}} .
$$

Example 10.2. Suppose that $x_{0}=0$, i.e., $P$ is a point of order 2. Then

$$
m=\sqrt{a_{6}}, x_{1}=\sqrt[4]{a_{6}}, y_{1}=l \sqrt[4]{a_{6}}+\sqrt{a_{6}} .
$$

If, in addition, $a_{2}=0$, then $l=0$ or 1 , and we get two halves $Q_{1}=\left(\sqrt[4]{a_{6}}, \sqrt{a_{6}}\right)$ and $Q_{1}=\left(\sqrt[4]{a_{6}}, \sqrt[4]{a_{6}}+\sqrt{a_{6}}\right)$ of $P$. This implies that $Q_{1}$ and $Q_{2}$ are (the only) points of order 4 .

The following assertion may be extracted from [4, Prop. 1.1].

Theorem 10.3. Suppose that $E(K)$ contains a point of order 2, i.e., there exists $\beta \in K$ such that $a_{6}=\beta^{2}$. Then a point $P=\left(x_{0}, y_{0}\right) \in E(K)$ is divisible by 2 in $E(K)$ if and only if the following conditions hold.

(i) $x_{0}$ is a square in $K$, i.e., there exists $r \in K$ such that $r^{2}=x_{0}$.

(ii) There exists $l \in K$ such that

$$
l^{2}+l=x_{0}+a_{2} .
$$

(iii) If $x_{0}=0$, i.e., $P$ is a point of order 2 , then $a_{6}$ is a fourth power in $K$.

Proof. Let $P=\left(x_{0}, y_{0}\right)$ is a $K$-point on $E$.

Assume that $P \in 2 E(K)$, i.e., there exists a point $Q=\left(x_{1}, y_{1}\right)$ on $E$ such that $2 Q=P$ and $x_{1}, y_{1} \in K$. Then in the notation above $l, m \in K$, because the tangent line to $E$ at $K$-point $Q$ is defined over $K$. It follows that (ii) holds. If $x_{0}=0$, then it follows from Example 10.2 that conditions (i)-(iii) hold. So, we may assume that $x_{0} \neq 0$. It follows from (34) that $\beta+m=x_{1} \sqrt{x_{0}}$. This implies that

$$
\sqrt{x_{0}}=\frac{\beta+m}{x_{1}}
$$

lies in $K$, i.e., (i) holds.

Now assume that conditions (i)-(iii) hold and $Q=\left(x_{1}, y_{1}\right) \in E(\bar{K})$ satisfies $2 Q=$ $P$. If $x_{0}=0$, then the explicit formulas of Example 10.2 tell us that $Q \in E(K)$. Suppose that $x_{0} \neq 0$. Let $\mathcal{L}: y=l x+m$ be the equation of the tangent equation to $E$ at $Q$. The condition (ii) implies that $l \in K$. Since $\mathcal{L}$ contains the $K$-point $-P, m$ also lies in $K$. Now equation (34) tells us that $\beta+m=x_{1} r$ with nonzero $r=\sqrt{x_{0}} \in K$, and therefore

$$
x_{1}=\frac{\beta+m}{r}
$$

also lies in $K$. This implies that $y_{1}=l x_{1}+m$ also lies in $K$. 
Corollary 10.4. An elliptic curve over a field $K$ of characteristic 2 has a $K$ rational point of order 4 if and only if there exists a nonzero $\gamma \in K$ such that $E$ is $K$-isomorphic to the elliptic curve

$$
\mathbf{E}_{4, \gamma}: y^{2}+x y=x^{3}+\gamma^{4} .
$$

Proof. The result follows almost immediately from Theorem 10.3 combined with Example 10.2. Indeed, if an elliptic curve $E$ over $K$ has a $K$-point of order 4, then it has a $K$-point of order 2 and therefore is ordinary, i.e., is $K$-isomorphic to

$$
y^{2}+x y=x^{3}+a_{2} x^{2}+a_{6},
$$

where

$$
a_{2}, a_{6} \in K ; a_{6} \neq 0, j(E)=\frac{1}{a_{6}} \neq 0
$$

and $a_{6}$ is a square in $K$. In addition, $P=\left(0, \sqrt{a_{6}}\right) \in E(K)$ is a point of order 2 that actually lies in $2 E(K)$. It follows from Theorem 10.3 that there exist $\beta, l \in K$ such that

$$
\beta^{4}=a_{6}, l^{2}+l=a_{2} .
$$

One should only notice that the change of variables $x \mapsto x, y \mapsto y+l x$ establishes a $K$-isomorphism between elliptic curves $y^{2}+x y=x^{3}+a_{2} x^{2} a_{6}$ and

$$
y^{2}+x y=x^{3}+\left(a_{2}+l^{2}+l\right) x^{2}+a_{6}=x^{3}+\beta^{4} .
$$

Corollary 10.5. Let $E$ be an elliptic curve over $K$ such that $E(K)$ contains a point of order 4 . Then $j(E)$ is a nonzero fourth power in $K$.

Conversely, if $c$ is a nonzero fourth power in $F$, then there exists an elliptic curve $E$ over $K$ such that $j(E)=c$ and $E(K)$ contains a point of order 4 . Such an $E$ is unique up to $K$-isomorphism.

Proof. Suppose $E(K)$ contains a point of order 4. By Corollary 10.4, there exists a nonzero $\gamma \in K$ such that $E$ is $K$-isomorphic to

$$
\mathbf{E}_{4, \gamma}: y^{2}+x y=x^{3}+\gamma^{4} .
$$

Clearly,

$$
j(E)=j\left(\mathbf{E}_{4, \gamma}\right)=\frac{1}{\gamma^{4}}=\left(\frac{1}{\gamma}\right)^{4} .
$$

This proves the first assertion.

Conversely, if $\delta \in K$ satisfies $\delta^{4}=c$, then we put $\gamma=1 / \delta$ and consider the elliptic curve $\mathbf{E}_{4, \gamma}$ over $K$. Recall that $\mathbf{E}_{4, \gamma}(K)$ contains a point of order 4 and

$$
j\left(\mathbf{E}_{4, \gamma}\right)=\frac{1}{\gamma^{4}}=\left(\frac{1}{\gamma}\right)^{4}=\delta^{4}=c .
$$

This proves the second assertion. In order to prove the uniqueness, let us assume that $E$ is an elliptic curve over $K$ such that $j(E)=c$ and $E(K)$ contains a point of order 4. Then, thanks to Corollary 10.4 there exists nonzero $\gamma \in K$ such that $E$ is $K$-isomorphic to $\mathcal{E}_{4, \gamma}$. This implies that

$$
c=j(E)=j\left(\mathbf{E}_{4, \gamma}\right)=\frac{1}{\gamma^{4}} .
$$

It follows that $\gamma=\sqrt[4]{1 / c}$. 
Theorem 10.6. An elliptic curve over a field $K$ of characteristic 2 has a $K$ rational point of order 8 if and only if there exists $t \in K \backslash\{0,1\}$ such that $E$ is $K$-isomorphic to the elliptic curve

$$
\mathbf{E}_{8, t}: y^{2}+x y=x^{3}+\left(\frac{t}{t^{2}+1}\right)^{8} .
$$

Proof. If an elliptic curve $E$ over $K$ has a $K$-point of order 8, then it has a $K$-point of order 4 , and therefore by Corollary 10.4 is $K$-isomorphic to

$$
\mathbf{E}_{4, \gamma}: y^{2}+x y=x^{3}+\gamma^{4},
$$

where $\gamma$ is a nonzero element of $K$. A point $Q$ of order 4 in $E(K)$ has $x$-coordinate $\gamma \neq 0$. Since $Q$ is divisible by 2 in $E(K)$, Theorem 10.3 tells us that there exist $l, r \in K$ such that

$$
l^{2}+l=\gamma=r^{2}
$$

Now the parametrization

of the conic

$$
l=\frac{1}{t^{2}+1}, r=\frac{t}{t^{2}+1}
$$

$$
l^{2}+l=r^{2}
$$

gives us the formula

$$
\gamma=\left(\frac{t}{t^{2}+1}\right)^{2}
$$

Remark 10.7. Let $t, s$ be two nonzero elements of $F$. Then

$$
t+\frac{1}{t}=s+\frac{1}{s}
$$

if and only if $s=t$ or $s=t^{-1}$. This implies that $\mathbf{E}_{8, t}=\mathbf{E}_{8, s}$ if and only if $s=t^{ \pm 1}$. (Hereafter both $s$ and $t$ are different from 1.) On the other hand,

$$
j\left(\mathbf{E}_{8, t}\right)=\left(t+\frac{1}{t}\right)^{-8}, j\left(\mathbf{E}_{8, s}\right)=\left(s+\frac{1}{s}\right)^{-8} .
$$

This implies that $\mathbf{E}_{8, t}$ and $\mathbf{E}_{8, s}$ are isomorphic over $\bar{K}$ if and only if $s=t^{ \pm 1}$, i.e., if and only if $\mathbf{E}_{8, t}=\mathbf{E}_{8, s}$.

Remark 10.8. Let us find explicitly a point of order 8 in $\mathbf{E}_{8, t}(K)$. We have the order 4 point

$$
P=\left(x_{0}, y_{0}\right)=\left(\gamma, \gamma^{2}\right) \in \mathbf{E}_{8, t}(K)
$$

and the equalities

$l=\frac{1}{t^{2}+1}, r=\frac{t}{t^{2}+1}, x_{0}=\gamma=\left(\frac{t}{t^{2}+1}\right)^{2}=l^{2}+l=r^{2}, y_{0}=\gamma^{2}=\left(\frac{t}{t^{2}+1}\right)^{4}$.

Let $Q=\left(x_{1}, y_{1}\right) \in \mathbf{E}_{8, t}(K)$ satisfy $2 Q=P$ and $l$ be the slope of the tangent line $y=l x+m$ to $E$ at $Q$. Then

$$
\left(y_{0}+x_{0}\right)-l x_{0}=m=x_{1}^{2} .
$$

This implies that

$$
\left(\gamma^{2}+\gamma\right)-\frac{\gamma}{t^{2}+1}=m=x_{1}^{2}
$$


i.e.,

$$
\begin{gathered}
x_{1}=(\gamma+\sqrt{\gamma})+\frac{\sqrt{\gamma}}{t+1}=\left(\frac{t}{t^{2}+1}\right)^{2}+\frac{t}{t^{2}+1}+\frac{t}{(t+1)^{3}}= \\
\frac{t^{2}+t\left(t^{2}+1\right)+t(t+1)}{(t+1)^{4}}=\frac{t^{3}}{(t+1)^{4}} .
\end{gathered}
$$

We have

$$
\begin{aligned}
& m=x_{1}^{2}=\frac{t^{6}}{(t+1)^{8}}, y_{1}=l x_{1}+m=\frac{1}{t^{2}+1} \frac{t^{3}}{(t+1)^{4}}+\frac{t^{6}}{(t+1)^{8}}=\frac{t^{3}}{(t+1)^{6}}+\frac{t^{6}}{(t+1)^{8}}= \\
& \frac{t^{3}\left(t^{2}+1\right)+t^{6}}{(t+1)^{8}}
\end{aligned}
$$

To summarize:

$$
Q=\left(\frac{t^{3}}{(t+1)^{4}}, \frac{t^{6}+t^{5}+t^{3}}{(t+1)^{8}}\right) \in \mathcal{E}_{8, t}(K)
$$

is a point of order 8 .

Corollary 10.9. Let $q$ be a power of 2 and $\mathbb{F}_{q}$ a finite field that consists of $q$ elements. Let $N$ be a power of 2 and $\Sigma(q, N)$ the set of $\mathbb{F}_{q}$-isomorphism classes of elliptic curves $E$ over $\mathbb{F}_{q}$ such that $E\left(\mathbb{F}_{q}\right)$ contains a point of order $N$. Then

$$
|\Sigma(q, 4)|=q-1,|\Sigma(q, 8)|=\frac{q}{2}-1 .
$$

Proof. We have $j\left(\mathbf{E}_{4, \gamma}\right)=1 / \gamma^{4}$. Since $\gamma$ may take any nonzero value in $\mathbb{F}_{q}$, it follows from Corollary 10.4 that $|\Sigma(q, 4)|=q-1$.

It follows from Theorem 10.6 combined with Remark 10.7 that there is a bijection between $\Sigma(q, 8)$ and the set of all unordered pairs $\left\{\left(t, t^{-1}\right) \mid t \in \mathbb{F}_{q} \backslash\{0,1\}\right\}$. Since $t \neq t^{-1}$ for each $t \neq 0,1$, the set of such pairs consists of $(q-2) / 2$ elements.

Example 10.10. Let us take $q=4$ and $K=\mathbb{F}_{4}$. Then there is exactly one elliptic curve $E$ over $\mathbb{F}_{4}$ (up to an $\overline{\mathbb{F}}_{4}$-isomorphism) such that $E\left(\mathbb{F}_{4}\right)$ contains a point of order 4 , namely,

$$
\mathbf{E}_{4, \rho}=\mathbf{E}_{4, \rho+1}: y^{2}+x y=x^{3}+1,
$$

where $\rho \in \mathbb{F}_{4} \backslash \mathbb{F}_{2}$ satisfies

$$
\rho^{2}+\rho+1=0, \rho^{3}=(\rho+1)^{3}=1, \rho^{-1}=\rho+1, \rho+\rho^{-1}=1 .
$$

The group $\mathbf{E}_{4, \rho}\left(\mathbb{F}_{4}\right)$ contains a point $Q$ of order 8, namely (Remark 10.8),

$$
Q=\left(\frac{\rho^{3}}{(\rho+1)^{4}}, \frac{\rho^{6}+\rho^{5}+\rho^{3}}{(\rho+1)^{8}}\right)=(\rho, \rho) .
$$

This implies that the order of the finite group $\mathbf{E}_{4, \rho}\left(\mathbb{F}_{4}\right)$ is divisible by 8 . On the other hand, the Hasse bound tells us that the order of $\mathbf{E}_{4, \rho}\left(\mathbb{F}_{4}\right)$ does not exceed $4+2 \sqrt{4}+1<8 \cdot 2$. This implies that $\mathbf{E}_{4, \rho}\left(\mathbb{F}_{4}\right)$ has order 8 and therefore coincides with its cyclic subgroup of order 8 generated by $Q$. 
FAMILIES OF ELLIPTIC CURVES WITH RATIONAL TORSION POINTS OF EVEN ORDEB1

\section{REFERENCES}

[1] B.M. Bekker, Yu.G. Zarhin, Division by 2 on elliptic curves. Algebra and Analiz 29:4 (2017), 196-239; arXiv:1702.02255 [math.NT].

[2] J.W.C. Cassels, Diophantine equations with special reference to elliptic curves. J. London Math. Soc. 41 (1966), 193-291.

[3] A. Knapp, Elliptic Curves. Mathematical Notes 40, Princeton University Press, Princeton, NJ, 1992.

[4] K. Kramer, Two-descent for elliptic curves in characteristic 2. Trans. Amer. Math. Soc. 232 (1977), 279-295.

[5] D.S. Kubert, Universal bounds on the torsion of elliptic curves. Proc. London Math. Soc. (3) 33 (1976), 193-237.

[6] E. Schaefer, 2-descent on the Jacobians of hyperelliptic curves. J. Number Theory 51 (1995), no. 2, 219-232.

[7] N. Schappacher, R. Schoof, Beppo Levi and the arithmetic of elliptic curves. Mathematical Intelligencer 18 (1996), 57-69.

[8] A. Silverberg, Explicit Families of Elliptic Curves with Prescribed Mod $N$ representations, pp. 447-461. In: Modular Forms and Fermat's Last Theorem (G. Cornell, J.H. Silverman, G. Stevens, eds.) Springer-Verlag New York Inc., 1997.

[9] J.S. Silverman, Arithmetic of Elliptic Curves. Second edition. Graduate Texts in Mathematics 106, Springer, Dordrecht Heidelberg London New York, 2009.

[10] J. Tate, Algebraic Formulas in Arbitrary Characteristic. Appendix 1 to: S. Lang, Elliptic Functions, Second Edition. Springer-Verlag New York Inc., 1987.

[11] L.C. Washington, Elliptic Curves: Number Theory and Cryptography. Second edition. Chapman \& Hall/CRC Press, Boca Raton London New York, 2008.

St. Petersburg State University, Department of Mathematics and Mechanics, UniVersitetsky prospekt, 28, Peterhof, St. Petersburg, 198504, Russia

E-mail address: bekker.boris@gmail.com

Pennsylvania State University, Department of Mathematics, University Park, PA 16802, USA

E-mail address: zarhin@math.psu.edu 